University of Louisville

ThinkIR: The University of Louisville's Institutional Repository

$5-2017$

\title{
Burkholderia cenocepacia J2315-mediated destruction of Staphylococcus aureus NRS77 biofilms.
}

\author{
Rachel Thompson \\ University of Louisville
}

Follow this and additional works at: https://ir.library.louisville.edu/honors

Part of the Environmental Microbiology and Microbial Ecology Commons, Molecular Biology Commons, and the Pathogenic Microbiology Commons

\section{Recommended Citation}

Thompson, Rachel, "Burkholderia cenocepacia J2315-mediated destruction of Staphylococcus aureus NRS77 biofilms." (2017). College of Arts \& Sciences Senior Honors Theses. Paper 138.

http://doi.org/10.18297/honors/138

This Senior Honors Thesis is brought to you for free and open access by the College of Arts \& Sciences at ThinkIR: The University of Louisville's Institutional Repository. It has been accepted for inclusion in College of Arts \& Sciences Senior Honors Theses by an authorized administrator of ThinkIR: The University of Louisville's Institutional Repository. This title appears here courtesy of the author, who has retained all other copyrights. For more information, please contact thinkir@louisville.edu. 


\title{
Burkholderia cenocepacia $\mathbf{J} 2315$-mediated destruction of Staphylococcus aureus NRS77 biofilms
}

\author{
By \\ Rachel Thompson \\ Submitted in partial fulfillment of the requirements for Graduation summa cum laude \\ and for Graduation with Honors from the Department of Biology
}

University of Louisville

May, 2017 


\section{Table of Contents}

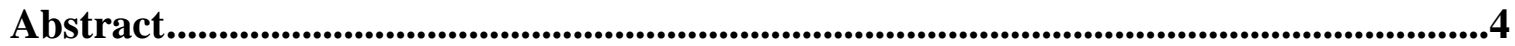

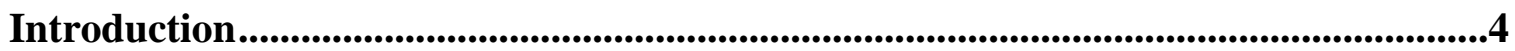

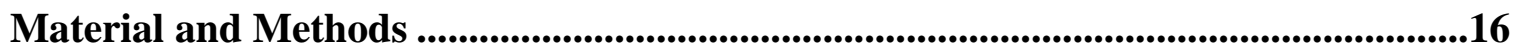

Bacterial Isolates and Materials .............................................................16

Biofilm Quantitation ............................................................................................17

Choosing Material for Optimized Biofilm Formation..........................17

Co-culture Biofilm Formation Assay Optimization.................................17

Sonication and Drip Dilutions............................................................18

Standardizing Viable Cell Count.................................................................19

Co-culture Biofilm Assays..........................................................................................20

Co-culture biofilms of five CF pathogens in pairwise combinations .............................................................................20

The effect of $B$. cenocepacia $\mathbf{J} 2315$ on multiple $S$. aureus

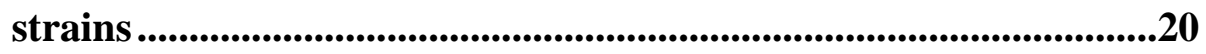

Pre-established $S$. aureus biofilm and B. cenocepacia $\mathrm{J} 2315$................21

Fractioning B. cenocepacia $\mathrm{J} 2315$ cultures...........................................23

Sonication of $\boldsymbol{B}$. cenocepacia $\mathbf{J} 2315$ cells and biofilm assay ................24

Phenotypes of Co-culture Biofilms ...............................................................................24

B. cenocepacia $\mathrm{J} 2315$ electrocompetent cells ..........................................25

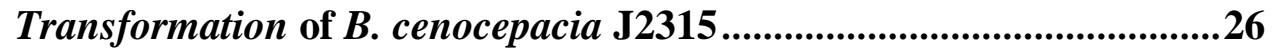

CLSM preparation and analysis.........................................................26

Statistical Analysis ...................................................................................27 
Results

Choosing Material for Optimized Biofilm Formation.......................................28

Co-Culture Biofilm Formation Assay Optimization .......................................28

Co-culture Biofilms of Five CF Pathogens in Pairwise Combinations ...........29

The effect of $\boldsymbol{B}$. cenocepacia $\mathbf{J} 2315$ on multiple $S$. aureus strain ....................30

Standardizing Viable Cell Count........................................................................33

Pre-established $S$. aureus biofilm and $B$. cenocepacia $\mathbf{J} 2315$........................34

Exploring the mechanism of $S$. aureus inhibition ............................................36

Transformation of B. cenocepacia J2315 ...............................................41

Phenotypes of Co-Culture Biofilms ..................................................................41

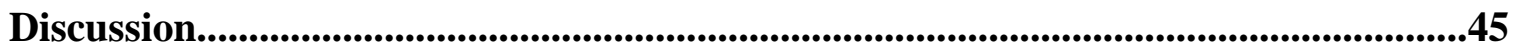

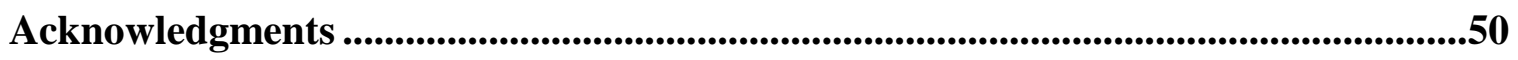

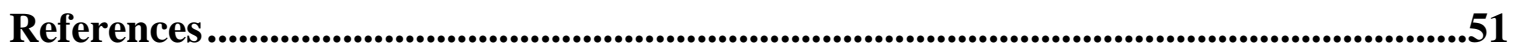

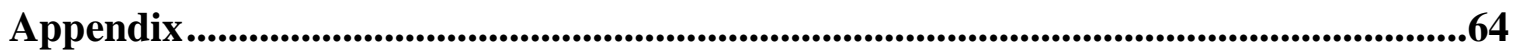

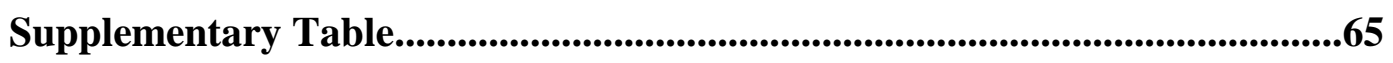

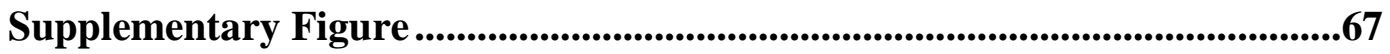




\section{Abstract}

Cystic fibrosis (CF) is an inherited disorder that affects over 30,000 people in the US and more than 70,000 people worldwide. Recurring bacterial infections in CF patients result in tissue damage that dramatically lowers respiratory function and are ultimately fatal. The formation of bacterial biofilms in the mucus and on lung epithelial tissue allows pathogens to be protected from antibiotics and the host immune system, making treatment of infection difficult. The interactions between $\mathrm{CF}$ pathogens in co-culture biofilms are not well understood and were examined in this study. Staphylococcus aureus and Burkholderia cenocepacia, two common CF pathogens, were used to examine the effects of co-culture on biofilm formation. We found that $S$. aureus biofilm formation and maintenance is inhibited when grown in co-culture with B. cenocepacia. In fact, supernatant from 3-day-old biofilms of B. cenocepacia was sufficient to reduce $S$. aureus biofilms, suggesting that a secreted compound may be responsible for this antagonism. The results of this study can be used to better understand the complex microenvironments bacteria experience in $\mathrm{CF}$ lungs. Further exploration of this interaction could lead to discovery of a novel therapeutic or a target for treatment of persistent $S$. aureus infection.

\section{Introduction}

\section{Cystic Fibrosis}

Cystic fibrosis (CF) is a recessive genetic disorder most common to Caucasians, a population in America in which 1 out of every 29 individuals is a carrier for a CF allele [1]. This disease is inherited by obtaining two copies of a mutant CFTR gene, which 
normally encodes an ion channel in the cell membrane called the cystic fibrosis transmembrane conductance regulator (CFTR). Both chloride and bicarbonate ions have been shown to utilize this protein to exit the cell, and they contribute to the maintenance of salt balance and mucin dissolution for healthy exocrine mucosal secretions [2].

Many different CFTR mutations can lead to a mutant phenotype [3], which can consist of either the absence or dysfunction of the CFTR channel. Due to CFTR defects, CF patients have a lowered ability or incapacity to transport chloride and bicarbonate anions outside of the cell [4]. The resulting ion imbalance causes dehydration of the mucus outside of the cell, hindering mucociliary clearance and making clearance through coughing arduous [5]. The altered mucus can affect the antimicrobial function of defensins, lysozymes, and other host pathogen-targeting compounds [5-7]. The mucus inside the lumen is rich in carbon and other nutrients, which can be metabolized by microbes [8]. Thus, the environment in the lumen of the CF lung is conducive to bacterial growth and infection.

Mutations in the CFTR gene can also result in compromised cellular and immune function. CF lungs are in a hyper-inflammatory state due to chronic bacterial infections as well as the accumulation of misfolded and degraded proteins inside the cell. The cellular process of autophagy normally assists in removal of unwanted proteins and cytosolic debris through fusion of phagosomes with degradative lysosomes [9]. Defects in autophagy have been observed in CF-derived cell lines and have been shown to lead to increased inflammation in the lung airways [10-12]. Furthermore, airway epithelial cells and neutrophils lacking functional CFTR proteins have altered cytokine responses compared to wild-type cells [13-15], suggesting a possible impairment of immune 
responses to pathogens.

Due to the viscosity of CF mucus, epithelial cilia are weighed down and clearance of microbes is severely impeded [16]. This stagnant environment can result in microbial growth in the mucus and allow bacteria to anchor to lung tissue. Bacteria in this environment can form biofilms, which are mats of bacterial cells embedded in an extracellular polymeric substance (EPS) made of polysaccharides, proteins, and extracellular DNA. EPS forms a sticky matrix that protects the enclosed bacteria from antibiotics, desiccation, and predation by immune cells.

The ability of bacteria in biofilms to evade the immune system and resist even the strongest antibiotics contributes to chronic bacterial infections and other symptoms seen in $\mathrm{CF}$ patients $[17,18]$. Neutrophil and macrophage invasion to the site of infection leads to inflammation, which can chronically damage host cells and lead to tissue damage [19]. There is a decrease in surface area for oxygen exchange in the scar tissue that develops from chronic inflammation [20]. Chronic infection and accumulating tissue damage over the course of their lives cause CF patients to experience a slow suffocation until the severity of lung damage leads to death.

Even though the airway in CF patients is rife with nutrients, only some opportunistic bacterial pathogens succeed in this environment. The most common pathogens in CF patients are Staphylococcus aureus, Pseudomonas aeruginosa, Haemophilus influenzae, Stenotrophomonas maltophilia, and members of the Burkholderia cepacia complex, including Burkholderia cenocepacia [21]. Of these, S. aureus and $P$. aeruginosa are the dominant pathogens and can be annually cultured from $80 \%$ of $\mathrm{CF}$ patients. Infections by these organisms are difficult to treat due to intrinsic 
antibiotic resistance. In addition, these pathogens are frequently spread between patients nosocomially $[22,23]$. Development of new therapeutics against these bacteria could benefit both CF patients and hospitalized individuals worldwide.

\section{Staphylococcus aureus}

S. aureus is a Gram-positive, non-motile bacterial species first discovered in 1880 in a skin abscess by Sir Alexander Ogsten [24]. It is estimated that $30 \%$ of the human population carry $S$. aureus as a commensal organism [25], usually in the upper respiratory tract, and it can be found commensally on other mammals [26-34]. The genome of $S$. aureus consists of a single 2.8 megabase pair (Mbp or 1 million base pairs) chromosome and contains pathogenicity islands for a variety of toxins and other virulence factors [34]. Plasmids and several other mobile genetic elements encode antibiotic resistance genes in both methicillin sensitive (MSSA) and methicillin resistant (MRSA) S. aureus strains.

As an opportunistic pathogen, $S$. aureus can cause a variety of infections in susceptible hosts. This species is the most frequent infectious agent of infective endocarditis [35] and osteoarticular infection [36]. This species can cause pneumonia [37], infection of prosthetic joints [38-39], and it can form biofilms on catheters [40],

prosthetic heart valves [41], pacemakers [42] and other prosthetic devices [43]. Skin and soft tissue infections by $S$. aureus manifest as abscesses, cellulitis, boils, impetigo, and infection of wounds [44].

As a primarily extracellular pathogen, the Microbial Surface Components Recognizing Adhesive Matrix Molecules (MSCRAMM) are a critical component of $S$. 
aureus pathogenicity and contribute to the initial attachment to host cells $[53,54]$. These proteins are located on the surface of the cell, covalently bound to the peptidoglycan of the cell wall [55]. Components of $S$. aureus MSCRAMM include fibronectin binding proteins, clumping factors, and S. aureus protein A (SpA) [56-59]. Fibronectin binding proteins and clumping factors act as adhesins, anchoring the cells to the extracellular matrix of host tissue for colonization [53]. SpA is a protein unique to $S$. aureus and contains several immunoglobulin-binding domains [60]. The function of SpA is to bind the Fc portion of immunoglobulin $\mathrm{G}$. This interaction prevents $\mathrm{Fc}$ from being recognized by - and interacting with - phagocytic neutrophils and macrophages, thereby allowing $S$. aureus to avoid detection by the circulating antibodies and evade classical complement formation, opsonization, and phagocytosis [61].

The majority of $S$. aureus strains produce exotoxins that function as superantigens, which are potent mitogens that trigger massive proliferation of $\mathrm{T}$ cells and cytokine production [45]. This immune response can overwhelm the human body and lead to septic shock. Enterotoxins secreted by this species and other staphylococci quickly cause food poisoning when consumed from undercooked, unpasteurized, or handler contaminated foods [46, 47]. Another $S$. aureus toxin, called toxic shock syndrome toxin, is a causative agent of toxic shock syndrome [48].

Other toxins produced by $S$. aureus strains do not act as superantigens but directly target host cells for destruction. Leukotoxins produced and secreted by S. aureus act to form holes in the membrane of host leukocytes, upsetting the osmotic balance and resulting in cell lysis [49]. The leukotoxin $\gamma$-hemolysin targets red blood cells and can cause massive hemolysis [50], while the monomeric $\alpha$-toxin (or $\alpha$-hemolysin) is highly 
cytotoxic and polymerizes to form a water channel in host cell membranes [51]. This leukotoxin leads to apoptosis through activation of a caspase cascade [52]. These leukotoxins extend bacterial infection by eliminating predatory immune cells and enhancing invasion of host tissue.

In addition to producing adhesins for attachment to host tissue during initial colonization, $S$. aureus also produces factors that assist in cell-to-cell binding and biofilm formation. The intercellular adhesin (ica) locus is found in S. aureus and several other staphylococcal species and encodes enzymes necessary for the production of polysaccharide intercellular adhesin (PIA), a component of staphylococcal EPS [62]. The products of the ica locus are necessary for initiation of biofilm formation by assisting in intercellular attachment between cells. The ability of $S$. aureus to form biofilm and attach to biomedical implants contributes to its ability to cause sepsis and recurring infection in host tissue.

Difficulty in treatment of $S$. aureus infection is due to the extensive range of antibiotics to which both MSSA and MRSA strains have evolved resistance. Over $90 \%$ of $S$. aureus isolates encode $\beta$-lactamase, eliminating certain penicillins as useful antibiotics [63]. While penicillinase-resistant penicillins are available, they are usually limited to treatment of severe MSSA infection. After its introduction in 1961, strains resistant to methicillin - a penicillinase-resistant penicillin - quickly surfaced [64], and MRSA strains have rapidly increased in prevalence of infections over the past 50 years. In the CF community, MRSA infection has increased from $6.1 \%$ of patients in 2000 to $26 \%$ in 2015 [21].

The mecA gene in MRSA isolates is located on the staphylococcal cassette 
chromosome mec (SCCmec) and encodes the enzyme that confers resistance to methicillin, penicillin, and other $\beta$-lactams [65]. The SCCmec is a mobile genetic element capable of horizontal gene transfer between staphylococcal strains [66]. Some studies suggest vancomycin is inferior to $\beta$-lactams as an antimicrobial, but it is still able to effectively eliminate infection of both MSSA and MRSA [67, 68]. However, strains bearing vancomycin resistance (VRSA) have also begun to appear in isolates of S. aureus [69], increasing the vital need for discovery of new anti-staphylococcal compounds and vaccine development for $S$. aureus.

S. aureus is typically the first organism that colonizes the CF lung and remains the most common $\mathrm{CF}$ isolate in individuals under 25, with prevalence of $S$. aureus colonization peaking between ages 11-15 [63,70]. Rates of MRSA infection during childhood are substantially higher in the United States than in Europe [70]. Approximately $25 \%$ of annual CF cultures are positive for MRSA, while the prevalence of MRSA in Europe remains about 5\%. This indicates that MRSA is a pressing problem in the U.S. and illustrates the need for the development of new treatments for MSSA and MRSA infection.

\section{Burkholderia cenocepacia}

The Burkholderia cepacia complex (Bcc) consists of 18 related Gram-negative species that thrive in a diverse array of environmental niches and have a broad array of metabolic capabilities, including nitrogen fixation [71], pollutant metabolism [72, 73], antifungal production [74], and plant pathogenesis [75]. These bacteria are typically found in the soil or water, and this versatility seen in Bcc ecological specialization is 
attributable to their uniquely complex genomes. Most Bcc species have genomes over twice the size of the Escherichia coli genome [76]. Where as the majority of bacteria have one circular chromosome, Bcc genomes consist of two or three circular chromosomes and at least one large plasmid [77]. The CF isolate B. cenocepacia J2315, for example, has four replicons, with approximate sizes of $3.8 \mathrm{Mbp}, 3.2 \mathrm{Mbp}, 875$ kilobase pairs $(\mathrm{kbp})$ and $92 \mathrm{kbp}$, which together encode over 7,000 predicted coding regions.

The first isolation of a Bcc member from a CF patient occurred in the 1970s [78], and a CF epidemic in the 1980s involving B. cenocepacia increased the significance of the Bcc as opportunistic human pathogens [79]. Analysis of the B. cenocepacia J2315 genome has implicated the role of horizontal gene transfer of genomic islands in the development of human virulence factors [77]. Currently, B. cenocepacia and $B$. multivorans are the most common Bcc species isolated from CF patients in North America [80] and Europe [81], respectively.

B. cenocepacia strains found in either the environment or as clinical isolates can be categorized into four different subgroups [82]. Subgroup IIIA includes B. cenocepacia J2315 and the ET12 lineage from which it is derived. The ET12 lineage of $B$. cenocepacia caused a large epidemic in the CF community in the 1980s and 1990s [83]. This strain was transmitted across the United Kingdom, Canada, and Europe due to contact between patients. The ET12 epidemic lineage encodes a cable pilus that can assist in binding to both mucins [84] and host tissue [85], and isolates have been shown to dominate and replace infections originally caused by B. multivorans [86].

B. cenocepacia J2315 contains genes that confer natural resistance to several 
antibiotics, including $\beta$-lactams, aminoglycosides, chloramphenicol, trimethoprimsulfamethoxazole, and gentamicin [77]. This strain also expresses type IV pili that are involved in motility and cellular adhesion to host tissue. Additionally, these pili have DNA binding activity [87] suggesting they may play a role in biofilm formation, in which extracellular DNA is a critical component of the secreted matrix. Like S. aureus, the ability of this strain to bind to both host tissue and form biofilms contributes to its virulence as a CF pathogen.

The secretion systems utilized by B. cenocepacia $\mathbf{J} 2315$ play an important role in the infection of human tissue. When cells of this strain are taken up by macrophages, the bacteria evade degradation and survive inside an endocytic vacuole [88]. The type IV secretion system has been implicated in the ability of B. cenocepacia to survive macrophage phagocytosis by evasion of lysosomal fusion [89]. The type VI secretion system has been shown to reduce production of reactive oxygen species inside macrophages [90].

The importance of Bcc as CF pathogens lie in their high transmissibility between patients and the quick progression of CF pathogenesis associated with Bcc infection [91]. "Cepacia syndrome" is characterized by bacteremia and a sudden rapid deterioration of lung function from necrotizing pneumonia [92]. The large majority of incidents of these symptoms occurred when CF patients were infected with a Bcc member. In addition, presence of Bcc is correlated with an increased risk of death in CF patients. Even with the dominance of $S$. aureus and $P$. aeruginosa as the most common CF pathogens, the risks of Bcc infection make exploration of their virulence necessary for the CF community. 


\section{Bacterial Biofilms}

Bacteria form biofilms as a defensive mechanism against a variety of potentially damaging conditions, such as presence of phagocytic predator cells, abrasive mechanical stimuli, or exposure to antibiotics. Initiation of biofilm formation involves quorum sensing, which is a method of altering gene expression at the population level based on cell density [93]. Initially, cells use adhesins and extracellular structures to reversibly attach to a surface, such as host tissue or synthetic material. During the second stage of biofilm formation, cells begin secreting molecules for formation of the EPS, which results in the irreversible attachment of bacteria to other cells and surfaces.

After permanent attachment to a surface, biofilm cells will begin to replicate, resulting in formation of clusters of bacteria termed microcolonies. Replication and EPS production continue until a mature biofilm with a unique three-dimensional structure is formed. Depending on how bacteria are communicating within or between species, different biofilm morphologies can be observed. P. aeruginosa, one of the most wellstudied biofilm formers, typically forms globular biofilms that resemble mushrooms [94]. This mushroom model of biofilm morphology is a commonly observed phenotype [95].

After biofilm maturation, the final stage of biofilm formation is the release of cells back to a planktonic, free-swimming lifestyle. This change involves dissolution of a portion of the biofilm EPS to allow cells to leave the biofilm and potentially begin the cycle all over again, initiating biofilm formation on another surface [96]. The biofilm lifestyle can increase the minimum inhibitory concentration of antibiotics 1000-fold [97,98] and make phagocytosis by macrophages difficult $[99,100]$, thus increasing the virulence and persistence of human bacterial pathogens inside the host. 


\section{Co-Culture Biofilms}

Single and polymicrobial biofilms can take on unique structures and spatial organizations that result in complex microenvironments [101, 102]. Different species can lay down in layers like stacks of paper, can arrange in a heterogeneous mixture of bacteria and involve contact with other species, or can remain in localized clusters with little to no cell-to-cell contact between microcolonies. Bacteria at the surface of a biofilm or in the lining of water channels will have different nutrient and oxygen availability than those near the bottom or the center of the biofilm. This arrangement results in unique metabolic states and requirements based on an organism's location within the biofilm.

Co-infection by pathogens in CF lungs is a common occurrence. In one group of CF patients participating in a clinical trial, $43.2 \%$ of individuals that were colonized by $P$. aeruginosa were also colonized by S. aureus [103]. Of 595 patients sampled, an average of 2.9 strains were cultured per person, including isolates of the biofilm-forming bacteria P. aeruginosa [104], Achromobacter xylosoxidans [105], B. cepacia [106], S. aureus [107], and S. maltophilia [108]. Given co-infection of CF patients with these bacteria, the CF lung likely acts as an ideal environment for the formation of mixed species biofilms, and the combinations of different strains may result in unique symptoms and severity of disease.

It is clear that a complex polymicrobial community inhabits CF lungs and that the microbial contents of CF airways change throughout a patient's lifetime. Multispecies biofilms and $\mathrm{CF}$ pathogen interactions are therefore important areas of research to understand CF pathogenesis. Monospecies biofilms have been studied in depth, such as those of $P$. aeruginosa $[93,109,110]$, but only a few studies have explored co-culture 
biofilms of CF pathogens. For example, the co-culture biofilm of $P$. aeruginosa and $S$. maltophilia may act to enhance antibiotic resistance compared to single species biofilms [111]. Clinical isolates of B. cepacia and $P$. aeruginosa grown in co-culture have been shown to form stable mixed biofilms [112]. In another study, P. aeruginosa PAO1 enhanced biofilm formation in S. aureus when grown in co-culture [113]. These studies are preliminary, and the interactions between many different $\mathrm{CF}$ pathogens remain to be explored. Currently, there is little to no understanding of the interaction between $S$. aureus and B. cenocepacia in a multispecies biofilm. The prevalence of the former and the mortality of the later make interactions between the pair an interesting topic of study.

The purpose of this study was to analyze polymicrobial interactions and biofilm formation in co-culture conditions of different CF pathogens. Originally, representative strains of five CF pathogens commonly isolated from the CF lung were tested for coculture biofilm formation. These pathogens included P. aeruginosa, S. aureus, B. cenocepacia, A. xylosoxidans, and S. maltophilia. Pairwise combinations of these species were grown in co-culture conditions and analyzed for biofilm formation, and the unique interaction between S. aureus and B. cenocepacia was chosen for further exploration. The data suggests that B. cenocepacia inhibits biofilm formation and maintenance of $S$. aureus. Based on these preliminary data, the main hypotheses for this study were that (1) $S$. aureus biofilm formation would be inhibited when grown in co-culture with $B$. cenocepacia J2315, (2) mature monoculter $S$. aureus biofilm would be diminished when exposed to B. cenocepacia, and (3) destruction of S. aureus biofilm in the presence of $B$. cenocepacia would occur over time. 


\section{Materials and Methods}

\section{Bacterial Isolates and Materials}

Luria Broth Lennox formulation (LB) was used to make both agar plates and liquid culture medium. Unless specified otherwise, all bacterial strains were plated from freezer stocks on LB agar plates and grown at $37^{\circ} \mathrm{C}$ for $24-48 \mathrm{~h}$. For overnight cultures, single colonies were inoculated into $5 \mathrm{~mL} \mathrm{LB}$ and incubated at $37^{\circ} \mathrm{C}$ with aeration until the next day. Plates and broth were supplemented with antibiotics as indicated. All strains can be found in the Yoder-Himes Lab strain collection. A complete list of all bacterial isolates used during the course of this project are listed and described in Table 1.

Table 1. Strains used in this study.

\begin{tabular}{|c|c|c|c|c|}
\hline Strain & Description & $\begin{array}{l}\text { Antibiotic } \\
\text { Selection }\end{array}$ & $\begin{array}{c}\text { Yoder- } \\
\text { Himes Lab } \\
\text { Strain } \\
\end{array}$ & Source \\
\hline $\begin{array}{l}\text { B. cenocepacia } \\
\mathrm{J} 2315\end{array}$ & $\mathrm{CF}$ clinical isolate & - & $\begin{array}{c}\text { Bcc isolates } \\
\text { A1-4 }\end{array}$ & $\begin{array}{l}\text { Dr. James Tiedje } \\
\text { (Michigan State } \\
\text { University) }\end{array}$ \\
\hline $\begin{array}{l}\text { B. cenocepacia } \\
\mathrm{J} 2315-62\end{array}$ & $\begin{array}{l}\text { J2315 bearing plasmid } \\
\text { pIN62 encoding } \\
\text { dsRedExpress }\end{array}$ & $\begin{array}{c}\text { chloramphenicol } \\
100\end{array}$ & $16-171$ & This study \\
\hline $\begin{array}{l}\text { B. cenocepacia } \\
\mathrm{J} 2315-301\end{array}$ & $\begin{array}{l}\text { J2315 bearing plasmid } \\
\text { pIN301 encoding GFP }\end{array}$ & $\begin{array}{c}\text { chloramphenicol } \\
100 \\
\end{array}$ & $16-172$ & This study \\
\hline S. aureus NRS77 & Blood clinical isolate & - & 13-012 & $\begin{array}{c}\text { BEI Resources; also } \\
\text { known as NCTC } 8325 \\
\text { or PS47 }\end{array}$ \\
\hline S. aureus NRS72 & $\begin{array}{c}\text { Bacteremia clinical } \\
\text { isolate }\end{array}$ & - & $13-011$ & $\begin{array}{l}\text { BEI Resources; also } \\
\text { known as Sanger476 }\end{array}$ \\
\hline $\begin{array}{l}\text { S. aureus } \\
\text { AH } 2183\end{array}$ & Derivative of NRS77 & - & $16-082$ & $\begin{array}{l}\text { Dr. Alexander Horswill } \\
\text { (University of Iowa) }\end{array}$ \\
\hline $\begin{array}{l}\text { S. aureus } \\
\text { AH3865 }\end{array}$ & $\begin{array}{l}\text { RN4220* containing a } \\
\text { plasmid encoding } \\
\text { dsRedExpress }\end{array}$ & $\begin{array}{c}\text { chloramphenicol } \\
10\end{array}$ & $16-081$ & $\begin{array}{l}\text { Dr. Alexander Horswill } \\
\text { (University of Iowa) }\end{array}$ \\
\hline $\begin{array}{l}\text { S. aureus } \\
\text { AH1710 }\end{array}$ & $\begin{array}{l}\text { RN4220* containing a } \\
\text { plasmid encoding GFP }\end{array}$ & $\begin{array}{c}\text { chloramphenicol } \\
10\end{array}$ & $16-080$ & $\begin{array}{l}\text { Dr. Alexander Horswill } \\
\text { (University of Iowa) }\end{array}$ \\
\hline $\begin{array}{l}\text { A. xylosoxidans } \\
\text { AU19284 }\end{array}$ & CF clinical Isolate & - & $13-005$ & $\begin{array}{c}\text { Dr. John LiPuma } \\
\text { (University of } \\
\text { Michigan) }\end{array}$ \\
\hline $\begin{array}{l}\text { S. maltophilia } \\
\text { K279a }\end{array}$ & Blood clinical isolate & - & $\begin{array}{c}\text { HMS Bcc/Pa } \\
3\end{array}$ & $\begin{array}{l}\text { Dr. Nick Cianciotto } \\
\text { (Northwestern } \\
\text { University) }\end{array}$ \\
\hline
\end{tabular}




\begin{tabular}{|c|c|c|c|c|}
\hline $\begin{array}{l}\text { P. aeruginosa } \\
\text { C3719 }\end{array}$ & $\begin{array}{l}\text { CF clinical isolate; } \\
\text { small colony variant }\end{array}$ & - & $\begin{array}{c}\text { HMS Bcc/Pa } \\
1 \\
\mathrm{H} 8-9 \\
\end{array}$ & $\begin{array}{c}\text { Dr. Stephen Lory } \\
\text { (Harvard Medical } \\
\text { School) }\end{array}$ \\
\hline $\begin{array}{l}\text { E. coli } \mathrm{DH} 5 \alpha \\
\text { pIN62 }\end{array}$ & $\begin{array}{l}\text { DH5 } \alpha \text { containing a } \\
\text { plasmid encoding } \\
\text { dsRedExpress }\end{array}$ & $\begin{array}{l}\text { chloramphenicol } \\
30\end{array}$ & $16-0103$ & $\begin{array}{l}\text { Dr. Annette Vergunst } \\
\text { (University of } \\
\text { Montpelier, France) }\end{array}$ \\
\hline $\begin{array}{l}\text { E. coli DH5 } \alpha \\
\text { pIN301 }\end{array}$ & $\begin{array}{c}\text { DH5 } \alpha \text { containing a } \\
\text { plasmid pIN301 } \\
\text { encoding GFP }\end{array}$ & $\begin{array}{c}\text { chloramphenicol } \\
30\end{array}$ & $16-0105$ & $\begin{array}{l}\text { Dr. Annette Vergunst } \\
\text { (University of } \\
\text { Montpelier, France) }\end{array}$ \\
\hline $\begin{array}{l}\text { E. coli DH5 } \alpha \\
\text { pIN298 }\end{array}$ & $\begin{array}{l}\text { DH5 } \alpha \text { containing a } \\
\text { plasmid pIN298 } \\
\text { encoding mCherry }\end{array}$ & $\begin{array}{c}\text { chloramphenicol } \\
30\end{array}$ & $16-0106$ & $\begin{array}{l}\text { Dr. Annette Vergunst } \\
\text { (University of } \\
\text { Montpelier, France) }\end{array}$ \\
\hline
\end{tabular}

*Restriction deficient $S$. aureus strain RN4220 is defective in the restriction-modification system that targets foreign DNA for destruction, therefore enhancing genetic manipulation of $S$. aureus in the lab.

\section{Biofilm Quantitation}

\section{Choosing Material for Optimized Biofilm Formation}

Overnight cultures of $S$. aureus NRS77, B. cenocepacia J2315, A. xylosoxidans AU19284, P. aeruginosa C3719, and S. maltophilia K279a in LB were diluted 1:100 in TSB, and $100 \mu \mathrm{L}$ of each culture was added to triplicate wells of both a sterilized 96-well PVC plate (Costar, \#29442-384) and a 96-well polystyrene plate (Greiner Bio-One, \#655185). The PVC plate was covered with porous sealing film (VWR, \#60941-086), and the polystyrene plate was covered with the provided lid. Both plates were incubated at $37^{\circ} \mathrm{C}$ for $24 \mathrm{~h}$. Quantitation of each monoculture biofilm on both plates was completed using the microtiter dish biofilm formation assay published by O'Toole [114]. Absorbance was measured using a Tecan Sunrise plate reader with optical density at $595 \mathrm{~nm}$.

\section{$\underline{\text { Co-culture Biofilm Formation Assays and Optimization }}$}

S. aureus NRS77, B. cenocepacia J2315, A. xylosoxidans AU19284, $P$. aeruginosa C3719, and S. maltophilia K279a were grown overnight and diluted 1:100 in Tryptic Soy Broth (TSB). Equal volumes of each strain were added to centrifuge tubes in 
pairs, then vortexed for 5-6 seconds to thoroughly mix the cultures. Two 96-well PVC plates were sterilized using $70 \%$ ethanol and allowed to dry in a biosafety cabinet. To both plates, $100 \mu \mathrm{L}$ of mixed co-culture was added to three replicate wells. Positive controls consisted of triplicate wells with $100 \mu \mathrm{L}$ of diluted single cultures. Plain TSB served as a negative control. The plates were sealed using porous sealing film to allow oxygen to freely cross, then covered with aluminum foil to help prevent desiccation. The plates were placed in an acrylic tub lined with damp paper towels and incubated at $37^{\circ} \mathrm{C}$ for 24 or $72 \mathrm{~h}$.

\section{$\underline{\text { Sonication and Drip Dilutions }}$}

Sonication was used to quantitate the number of cells in a biofilm using a procedure adapted from Harrison, et al [115]. After the 96-well plate had been incubated for the desired amount of time, the foil and sealing film were removed and the liquid culture dumped into a $10 \%(\mathrm{v} / \mathrm{v})$ bleach solution. The plate was gently and briefly placed upside down on a paper towel to allow residual liquid to be removed from the surface of the plate. This step helps prevent cross contamination of the wells in the later steps of the procedure. Aliquots of $115 \mu \mathrm{L}$ of sterile water were added to the wells, and the samples were incubated for one min. The water was dumped out of the plate and $115 \mu \mathrm{L}$ of $1 \%$ Tween 20 in LB was added to each well to act as the recovery medium. The plate was covered with sealing foil (USA Scientific, \#2923-0110) and placed on the bottom level of a test tube rack. The rack was suspended inside an ultrasonic cleaner (Branson 3200) until the liquid in the wells was submerged below the level of the water in the cleaner. The plate was subjected to sonication at $60 \mathrm{~Hz}$ for $10 \mathrm{~min}$ to disrupt biofilms, as 
suggested in the protocol by Harrison, et al [115].

The foil was wiped down with $70 \%$ ethanol to ensure it was sterilized. A pipet tip was use to punch through the foil and transfer $20 \mu \mathrm{L}$ from the well to $180 \mu \mathrm{L}$ phosphate buffer solution (PBS) in row A of a separate flat bottom polystyrene 96-well plate. After

the entire row had been inoculated, 10-fold serial dilutions were performed. Using an eight-channel pipettor, $10 \mu \mathrm{L}$ of the entire serial dilution were transferred to a square plate containing LB medium, which was set on its side and slanted to allow the drops to run down the agar. The drops were allowed to run three quarters of the way down the plate before replacing the lid and setting the plate down flat.

Plates were incubated at $37^{\circ} \mathrm{C}$ for $24-48 \mathrm{~h}$, or until colonies became distinguishable. Supplementary Table 1 shows the colony morphology and incubation time for each strain. Typically, S. aureus colonies were counted after $24 \mathrm{~h}$, while $B$. cenocepacia colonies were counted after $48 \mathrm{~h}$. Colonies were counted in the drip line that contained between 11-100 colonies for each specific strain, and the number of live bacteria per milliliter of recovery medium (colony forming units per milliliter or $\mathrm{CFU} / \mathrm{mL}$ ) was calculated using both these values and the dilution factor of the most appropriate dilution.

\section{$\underline{\text { Standardizing Viable Cell Count }}$}

To eliminate the variability in the viable cell count of overnight cultures between strains, drip dilutions were performed for cultures grown to an optical density at $600 \mathrm{~nm}$ (O.D.600) of 0.8, which corresponds to mid-log phase growth. Overnight cultures were grown by inoculation of $5 \mathrm{~mL}$ LB with the desired strain, followed by incubation at $37^{\circ} \mathrm{C}$ 
with aeration until the next day. Overnight cultures were made for B. cenocepacia $\mathbf{J} 2315$, S. aureus NRS77, S. aureus NRS72, S. aureus AH3865, S. aureus AH2183, S. aureus AH1710, and S. aureus AH2547. For each strain, $100 \mu \mathrm{L}$ of the overnight culture was added to fresh, pre-warmed LB. These were allowed to grow at $37^{\circ} \mathrm{C}$ with aeration until O.D.600 reached 0.8, and the tubes were placed on ice until all strains reached this O.D. The cultures were serially diluted in PBS, and then drip dilutions were performed to quantitate the viable cell count of each strain at O.D.600 of 0.8 .

\section{Co-Culture Biofilm Assays}

\section{$\underline{\text { Co-culture Biofilms of five CF Pathogens in Pairwise Combinations }}$}

The methods described under the sections Co-culture Biofilm Formation Assays

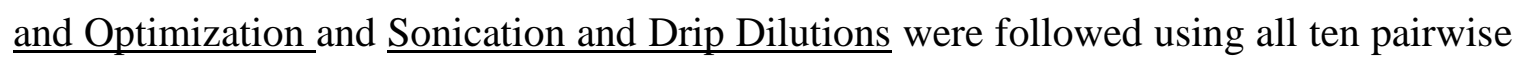
combinations of B. cenocepacia J2315, S. aureus NRS77, A. xylosoxidans AU19284, P. aeruginosa C3719, and S. maltophilia K279a. For each combination, equal volumes of cultures were added to microfuge tubes and vortexed for 5-6 seconds. Triplicate wells of two 96-well PVC plates received $100 \mu \mathrm{L}$ of each mixture and plates were incubated for 1 or 3 days. During drip dilutions, only the $10^{3}-10^{7}$ dilutions were plated. Plating of $10 \mu \mathrm{L}$

of this serial dilution therefore placed the limit of detection of the viable cell counts at $10^{5}$ $\mathrm{CFU} / \mathrm{mL}$.

The effect of $B$. cenocepacia $\mathrm{J} 2315$ on multiple $S$. aureus strains

To examine the ability of B. cenocepacia J2315 to affect various $S$. aureus strains, four S. aureus isolates were used and paired with B. cenocepacia J2315 in co-culture 
conditions. Diluted cultures of each S. aureus strain, NRS77, NRS72, AH2183, and AH3865 were mixed 1:1 (v/v) with diluted B. cenocepacia J2315 culture. In addition to each co-culture, monocultures were also inoculated in triplicate wells of three different 96-well PVC plates, which were incubated for 1, 3, or 7 days. Wells containing TSB acted as the negative control. The procedure for the co-culture biofilm formation assay, with sonication and drip dilutions, was followed for this experiment.

Inoculation of Pre-established S. aureus NRS77 Biofilms with B. cenocepacia J2315

S. aureus NRS77 was grown to an O.D.600 of 0.8, then diluted 1:10 in TSB. In a sterilized 96-well PVC plate, $100 \mu \mathrm{L}$ of the culture was added to 10 sets of triplicate wells. Another set of wells was inoculated with TSB as a negative control. The plate was sealed with porous film, covered with aluminum foil, and placed in an acrylic bin lined with damp paper towels. After covering the bin with plastic wrap, the bin was placed in a $37^{\circ} \mathrm{C}$ room for $72 \mathrm{~h}$. Water was added to the bin daily to prevent desiccation of the biofilms.

The same day that the $S$. aureus monoculture biofilm plate was to be removed, $B$. cenocepacia J2315 and S. aureus NRS77 cultures were grown to an O.D.600 of 0.8 then diluted 1:100 or 1:10 in TSB, respectively, in a microfuge tube. Based on the results from the Standardizing Viable Cell Count experiment in the section above, the dilutions of both strains had approximately the same cell count. Three different cultures were then made from these dilutions: B. cenocepacia J2315 monoculture, S. aureus NRS77 monoculture, and co-culture of B. cenocepacia J2315 and S. aureus NRS77. In addition to equalizing the cell count between strains, the total cell count of each strain must be the 
same in the co-culture condition as in the single culture condition. This feat was accomplished by aliquoting $1 \mathrm{~mL}$ of diluted B. cenocepacia $\mathrm{J} 2315$ culture into a microfuge tube, centrifugation of the cells for one min at $21,130 \times g$, pouring off the supernatant, then resuspending the pellet in $1 \mathrm{~mL}$ of the diluted $S$. aureus NRS77 culture. All microfuge tubes were vortexed for 6 seconds to homogenize the mixtures.

The covers from the established $S$. aureus biofilms were removed and the biofilm supernatant was poured into a bleach solution. After dabbing off excess liquid from the surface of the plate, $100 \mu \mathrm{L}$ of diluted S. aureus NRS77 was added to three sets of triplicate wells, one set each for day 1, 3 and 7. This step was repeated for the culture of diluted $B$. cenocepacia and the co-culture mixture. In addition, $100 \mu \mathrm{L}$ of diluted $B$. cenocepacia was added to three triplicate non-biofilm containing wells to act as a positive control for biofilm formation at the point of inoculation. TSB was used as a negative control.

At this point, 9 sets of the triplicate S. aureus NRS77 biofilm wells were inoculated with either experimental or control treatments. The tenth set of S. aureus biofilm wells and the negative control from initial $S$. aureus inoculation were washed with $115 \mu \mathrm{L}$ sterile water then scraped into $115 \mu \mathrm{L}$ of $1 \%$ Tween 20 recovery medium using a P200 pipette tip. The medium was pipetted out of the wells, serially diluted, and plated for drip dilutions to determine the starting $\mathrm{CFU} / \mathrm{mL}$ of the $S$. aureus biofilm at the point of B. cenocepacia inoculation.

The biofilms were once again covered with porous sealing film and aluminum foil, and then placed on damp paper towels in an acrylic, plastic-wrap-covered bin. The plate was incubated for 1, 3, and 7 days. On each day, the plate was uncovered, the liquid 
culture of the wells of interest were removed with a pipette, and the wells were washed with sterile water, as described above. Because wells containing conditions for 1,3 , and 7 day biofilm formation were all on the same plate, biofilms were disrupted by scraping with a $200 \mu \mathrm{L}$ pipette tip, instead of through sonication. Biofilms were quantitated using drip dilutions. The plate was covered and incubated at $37^{\circ} \mathrm{C}$ between time points. On the final day, the negative control was also plated.

\section{Fractionating B. cenocepacia $\mathrm{J} 2315$ cultures}

B. cenocepacia J2315 cultures were separated into the supernatant and cellular components. Supernatant was isolated in two ways. First, B. cenocepacia J2315 was grown in a biofilm for three days in 64 wells of a 96-well PVC plate, as described above for the growth of monoculture $S$. aureus biofilm. On day three, the liquid from each well was removed and added to a microfuge tube. The cells were pelleted at $21,130 \times \mathrm{x}$ in a microfuge for one min, and the supernatant was filter-sterilized through a $0.45 \mu \mathrm{m}$ syringe filter. Aliquots of this sterilized supernatant were plated to ensure no viable $B$. cenocepacia J2315 cells remained. The total volume of collected supernatant was approximately $6.4 \mathrm{~mL}$. To this, $6.4 \mathrm{~mL}$ of $20 \%$ glycerol in LB was added to result in a $10 \%$ glycerol solution that could be stored at $-20^{\circ} \mathrm{C}$ until use.

Overnight culture supernatant was also isolated. B. cenocepacia $\mathrm{J} 2315$ was grown overnight in LB, centrifuged at 21,130 x $g$ for one min, and the supernatant was filtersterilized. Each supernatant solution was added 1:1 (v/v) with TSB and added to $S$. aureus biofilm to yield a $25 \%$ biofilm supernatant treatment and a $50 \%$ overnight supernatant treatment, both with 50\% TSB. Additionally, the pellet formed after the 
centrifugation of the overnight B. cenocepacia $\mathrm{J} 2315$ culture was resuspended and diluted 1:100 in TSB before addition to S. aureus biofilms.

$\underline{\text { Sonication of } B \text {. cenocepacia } \mathrm{J} 2315 \text { cells and biofilm assay }}$

To determine if live cells were needed for the toxic effect on S. aureus, dead $B$. cenocepacia cells were made by placing an overnight culture of the J2315 strain in a $65^{\circ} \mathrm{C}$ water bath for 30 min then sonicated at $60 \%$ amplitude for 10 second pulses over a period of $10 \mathrm{~min}$ in a Qsonica cup sonicator (Qsonica, Q800R). This culture was not diluted, but mixed with TSB in a 1:1 (v/v) ratio. Plating of serial dilutions of the resulting culture showed no viable cells remained after sonication.

Live cells, sonicated cells, or either supernatants were added to triplicate wells containing one-day old S. aureus biofilms and also to empty wells to act as controls. One set of triplicate wells containing S. aureus biofilm received $100 \mu \mathrm{L}$ plain TSB to act as a control for $S$. aureus biofilm formation. Another set of empty wells received TSB as a negative control. Two PVC plates were made with these conditions, and one was incubated at $37^{\circ} \mathrm{C}$ for $24 \mathrm{~h}$, while the second was incubated for $72 \mathrm{~h}$. The protocol described above in Sonication and Drip Dilution Biofilm was used to quantitate biofilm formation.

\section{Phenotypes of Co-culture Biofilms}

$\underline{\text { Super Optimal Broth Preparation for B. cenocepacia Transformation }}$

Super optimal broth (SOB) and super optimal broth with catabolite repression (SOC) are media used to promote higher plasmid transformation efficiency in bacteria. 
To make SOB, $20 \mathrm{~g}$ tryptone, $5 \mathrm{~g}$ yeast extract, and $0.5 \mathrm{~g} \mathrm{NaCl}$ were added to $950 \mathrm{~mL}$ sterile water and shaken until dissolved. To this solution, $10 \mathrm{~mL}$ of $250 \mathrm{mM} \mathrm{KCl}$ was added and the $\mathrm{pH}$ adjusted to 7.0. After autoclaving for $20 \mathrm{~min}$, the medium was placed in a $55^{\circ} \mathrm{C}$ water bath to cool for about $30 \mathrm{~min}$. Gentamicin $(10 \mu \mathrm{g} / \mathrm{mL})$ was added to the medium to prevent contamination, as suggested by published protocol for transformation of B. cenocepacia J2315 [116]. Right before the medium was used, $10 \mathrm{~mL}$ of sterile $1 \mathrm{M}$ $\mathrm{MgSO}_{4}$ and $5 \mathrm{~mL} 2 \mathrm{M} \mathrm{MgCl}_{2}$ were added. SOC broth was made by adding $20 \mathrm{~mL}$ of a 1 M glucose solution to $1 \mathrm{~L} \mathrm{SOB}$.

\section{B. cenocepacia Electrocompetent Cells}

To create electrocompetent cells for transformations, an overnight culture of $B$. cenocepacia J2315 was grown in $5 \mathrm{~mL}$ SOB medium. The next day, the culture was diluted into an Erlenmeyer flask containing $50 \mathrm{~mL}$ SOB to make a final O.D.600 of 0.01. The flask was incubated with aeration at $37^{\circ} \mathrm{C}$ for $2-3$ generations, until the O.D.600 was between $0.04-0.08$ based on the published protocol [116]. This equates to about 4-6 h of incubation. Next, the flask was chilled on ice for 5 min to stop bacterial growth, and the culture was aliquoted and centrifuged at $5000 \times \mathrm{g}$ for $8 \mathrm{~min}$ at $4{ }^{\circ} \mathrm{C}$ (Kendro Lab Products, Sorvall Legend RT Centrifuge). The supernatant was removed, and the pellet was washed and resuspended in $20 \mathrm{~mL}$ ice-cold sterile $0.5 \mathrm{M}$ sucrose, followed by two additional rounds of centrifugation, washing, and resuspension. The final pellet was resuspended in $0.5 \mathrm{~mL}$ 0.5 M sucrose. An LB and glycerol solution was added to make the final concentration $10 \%$ glycerol. The sample was divided into $500 \mu \mathrm{L}$ aliquots and stored at $-80^{\circ} \mathrm{C}$ until electroporation. 


\section{Transformation of B. cenocepacia $\mathrm{J} 2315$}

Following the QIAprep Spin Miniprep Kit (Qiagen, \#27106) protocol, plasmids encoding fluorescent proteins were purified from the E. coli $\mathrm{DH} 5 \alpha$ strains provided by Dr. Annette Vergunst (University of Montpelier, France). These are listed in Table 2. The purity and DNA concentration for each purified plasmid was determined using a Nanodrop 2000 (Thermo Fisher Scientific). Plasmids were stored at $-20^{\circ} \mathrm{C}$ until use.

The plasmids, thawed electrocompetent $B$. cenocepacia cells, and electroporation cuvettes (VWR, \#89047-206) were kept on ice. To $15 \mu \mathrm{L}$ water, $0.1 \mu \mathrm{g}$ of each plasmid was added, followed by the addition of $55 \mu \mathrm{L}$ of electrocompetent $B$. cenocepacia. The negative control consisted of electrocompetent cells without the addition of plasmid. After transferring this volume to a $2 \mathrm{~mm}$ electroporation cuvette, cells were electroporated at $2.5 \mathrm{kV}$ (Bio-Rad Micropulser). Samples were removed from the cuvette by adding $950 \mu \mathrm{L}$ SOC medium, and after gentle mixing, the volume was transferred to a microfuge tube. Samples were incubated at $37^{\circ} \mathrm{C}$, without aeration, for $4-5 \mathrm{~h}$ and plated on LB plates supplemented with $100 \mu \mathrm{g} / \mathrm{mL}$ chloramphenicol. Plates were incubated for three days. Single colonies were plated in patches on selective plates, and the resulting growth was observed under a confocal microscope to confirm fluorescence. Colonies that showed the proper fluorescence profile were stored in $20 \%$ glycerol at $-80^{\circ} \mathrm{C}$ under the strain list designations shown in Table 1.

\section{Confocal Laser Scanning Microscopy Preparation and Analysis}

To capture images of both single and co-culture biofilms, the fluorescent strains $S$. aureus AH1710 and B. cenocepacia J2315-62 were utilized. Each strain was individually 
inoculated in test tubes containing $5 \mathrm{~mL}$ LB supplemented with $10 \mu \mathrm{g} / \mathrm{ml}$ or $100 \mu \mathrm{g} / \mathrm{ml}$ chloramphenicol, respectively. Cultures were incubated at $37^{\circ} \mathrm{C}$ overnight then diluted 1:100 in TSB supplemented with $10 \mu \mathrm{g} / \mathrm{mL}$ chloramphenicol. Glass bottom FluoroDishes (World Precision Instruments, FD35-100) were used to grow the biofilms. Three sets of triplicate dishes were made for each monoculture and co-culture conditions. Dishes contained $3 \mathrm{~mL}$ of $S$. aureus $\mathrm{AH} 1710,3 \mathrm{~mL}$ of B. cenocepacia $\mathrm{J} 2315-62$, or $3 \mathrm{~mL}$ of a 1:1 by volume mixture of the two. Confocal laser scanning microscopy (CLSM) analysis occurred at 1 day, 3 days, and 7 days post-inoculation of each condition. At each time point, the biofilm supernatant was discarded prior to microscopy. Both 2D and 3D imaging was captured using NIS-Element software with both the 40x and 60x lenses of a Nikon Eclipse Ti microscope. The GFP $(487 \mathrm{~nm})$ and TxRed $(561 \mathrm{~nm})$ lasers were used for excitation wavelengths. The power and gain of each image was optimized to show maximum fluorescence without saturation of the image.

\section{$\underline{\text { Statistical analyses }}$}

All biofilm assays and viable cell count assays were graphed and analyzed using GraphPad Prism v. 5.1.0. Statistical significance was calculated using either two-way ANOVA analysis, one-way ANOVA analysis in conjunction with Tukey's multiple comparison test, or an unpaired T test, as indicated in each figure legend. Only p-values less than 0.05 were considered statistically significant. 


\section{Results}

\section{Choosing Material for Optimized Biofilm Formation}

In order to test for the plate material that optimizes biofilm formation, representative strains of five common $\mathrm{CF}$ pathogens were chosen to form monoculture biofilms in 96 well plates made of two different materials. Twenty-four hour monoculture biofilms of $S$. aureus NRS77, B. cenocepacia J2315, A. xylosoxidans AU19284, P. aeruginosa C3719, and S. maltophilia K279a were grown in both round-bottom 96-well PVC plates and flat-bottom 96-well polystyrene plates. Biofilm was quantitated using the retention of crystal violet as described in the Materials and Methods. Results are shown in Figure 1.

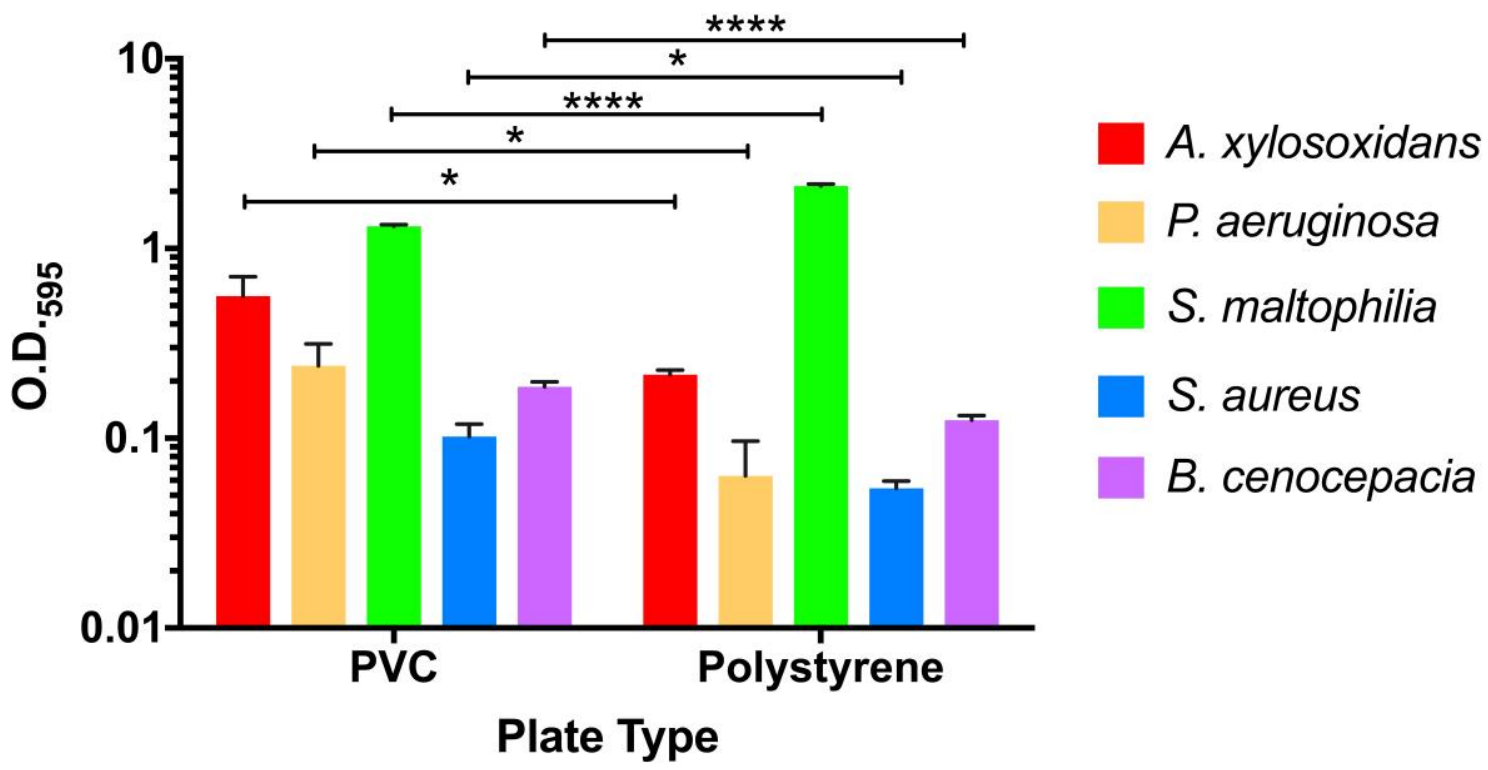

Figure 1. Biofilm formation in polystyrene and PVC 96-well plates. The optical density of biofilm growth was obtained for one-day-old monocultures of A. xylosoxidans AU19284 (red), $P$. aeruginosa C3719 (orange), S. maltophilia K279a (green), S. aureus NRS77 (blue), and $B$. cenocepacia J2315 (purple) grown in both PVC and polystyrene 96-well plates. Two-way ANOVA analysis showed an overall significant effect of the interaction between strain type and plate material $(\mathrm{p}<0.0001)$. Unpaired t-tests were used to calculate significance between strains grown on each plate material $(* \mathrm{p}<0.05, * * * * \mathrm{p}<0.0001)$. 
Unpaired t-tests of each strain showed that for all strains except $S$. maltophilia K279a, biofilm formation was significantly greater in 96-well PVC plates compared to polystyrene 96-well plates (Fig. 1). S. maltophilia K279a grew optimally in the polystyrene 96-well plate but was still able to form biofilm that was comparable to the biofilm formation of the other four isolates in the 96-well PVC plate. Due to these observations, the 96-well PVC plate was chosen to use in all future biofilm formation assays.

\section{Co-culture Biofilms of Five CF Pathogens in Pairwise Combinations}

After establishing that round-bottom, 96-well PVC plates were conducive for biofilm formation for a majority of the strains (Fig. 1), the same five isolates were tested for compatibility of biofilm formation in co-culture conditions. All pairwise combinations of B. cenocepacia J2315, S. aureus NRS77, A. xylosoxidans AU19284, P. aeruginosa C3719, and S. maltophilia K279a were inoculated in triplicate wells of a 96well PVC plate and incubated for 1 or 3 days. Biofilm quantitation was completed using sonication and drip dilutions, and viable cell counts for each strain in co-culture were compared to each strain in monoculture.

Supplementary Figure 1 shows the data and summarizes the results for monoculture and co-culture biofilm formation of A. xylosoxidans AU19284, $P$. aeruginosa C3719, and S. maltophilia K279a. The data for B. cenocepacia J2315 and S. aureus NRS77 (Fig. 2) suggested that the presence of S. aureus NRS77 has no effect on B. cenocepacia $\mathrm{J} 2315$ biofilm formation, but the presence of B. cenocepacia $\mathrm{J} 2315$ results in a reduction in viable cells below the limit of detection for S. aureus NRS77 
biofilm by day 3 . These results suggested that the presence of B. cenocepacia J2315 may inhibit $S$. aureus NRS77 biofilm formation over time.
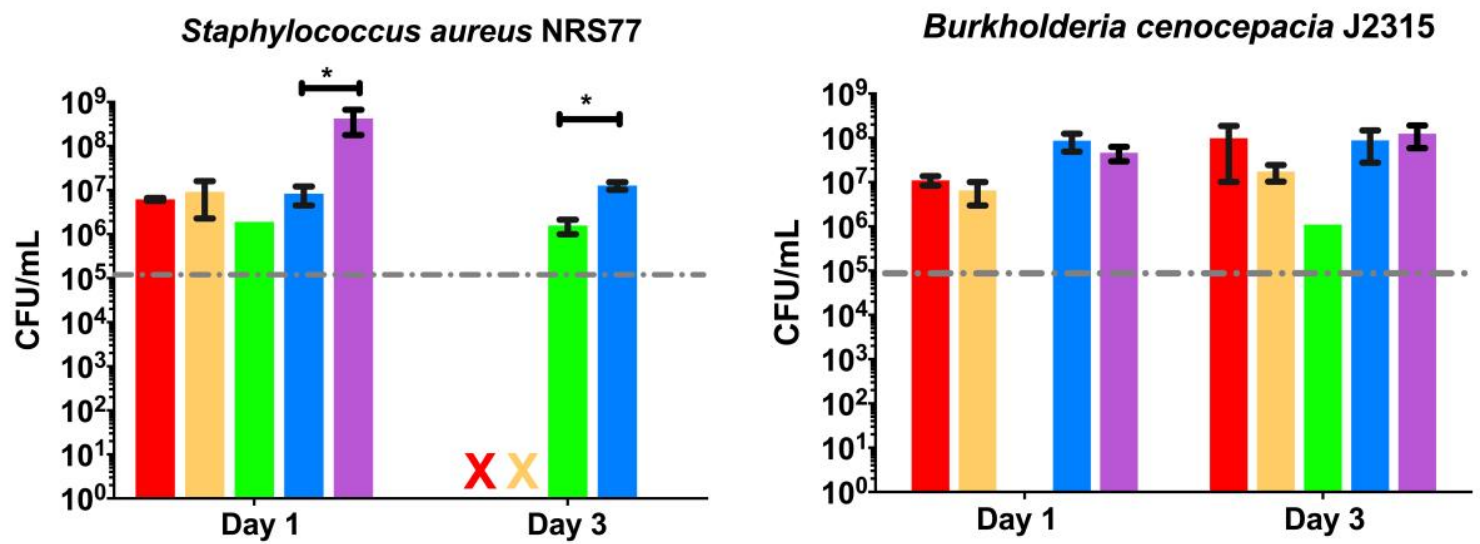

A. xylosoxidans 19284

P. aeruginosa C3719
S. maltophilia K279a

S. aureus NRS77

B. cenocepacia J2315

Figure 2. Biofilm formation of B. cenocepacia J2315 and $S$. aureus NRS77 in co-culture conditions. Viable cell counts of (A) S. aureus NRS77 biofilms and (B) B. cenocepacia J2315 biofilms when grown in co-culture with A. xylosoxidans (red), P. aeruginosa C3719 (orange), $S$. maltophilia K279a (green), S. aureus NRS77 (blue), or B. cenocepacia J2315 (purple). Presence of an "X" indicates exclusion of data due to contamination of samples. Absence of a bar indicates the viable counts are below the limit of detection (gray line), which was $10^{5}$ for this experiment. One-way ANOVA analysis showed an overall significant difference among means $(\mathrm{p}<0.05)$ in $\mathrm{CFU} / \mathrm{mL}$ within both $S$. aureus NRS77 and B. cenocepacia J2315 data. Tukey's multiple comparison test was used to compare co-culture and monoculture conditions $(* \mathrm{p}<0.05)$.

\section{Effect of B. cenocepacia J2315 on S. aureus Strains}

Next, the ability of $B$. cenocepacia J2315 to inhibit biofilm formation in other $S$. aureus strains was tested. It was hypothesized that inhibition of $S$. aureus biofilm by $B$. cenocepacia J2315 would not be limited to the NRS77 strain. Mixes of B. cenocepacia J2315 and four S. aureus strains - NRS77, NRS72, AH2183, and AH3865 - were made and inoculated in a 96-well PVC plate and allowed to incubate for 1, 3, or 7 days before the co-culture biofilms were quantitated by sonication and drip dilutions. The viable cell count was then compared to viable counts from monoculture biofilms. 
There was no statistical significance in biofilm formation of B. cenocepacia $\mathrm{J} 2315$ between S. aureus strains or over time (Fig. 3), while all tested S. aureus strains were inhibited over time by the presence of B. cenocepacia J2315 (Fig. 4). Viable S. aureus NRS77 biofilm was below the limit of detection $\left(10^{3} \mathrm{CFU} / \mathrm{mL}\right)$ in the presence of $B$. cenocepacia J2315 (Fig. 4A). Biofilms of S. aureus NRS72 were present after growing for 1 day with $B$. cenocepacia $\mathrm{J} 2315$, but growth was significantly inhibited in comparison to the monoculture biofilm, and by day 3, viable $S$. aureus NRS72 biofilm was reduced beyond the limit of detection in co-culture with B. cenocepacia J2315 (Fig. 4B).

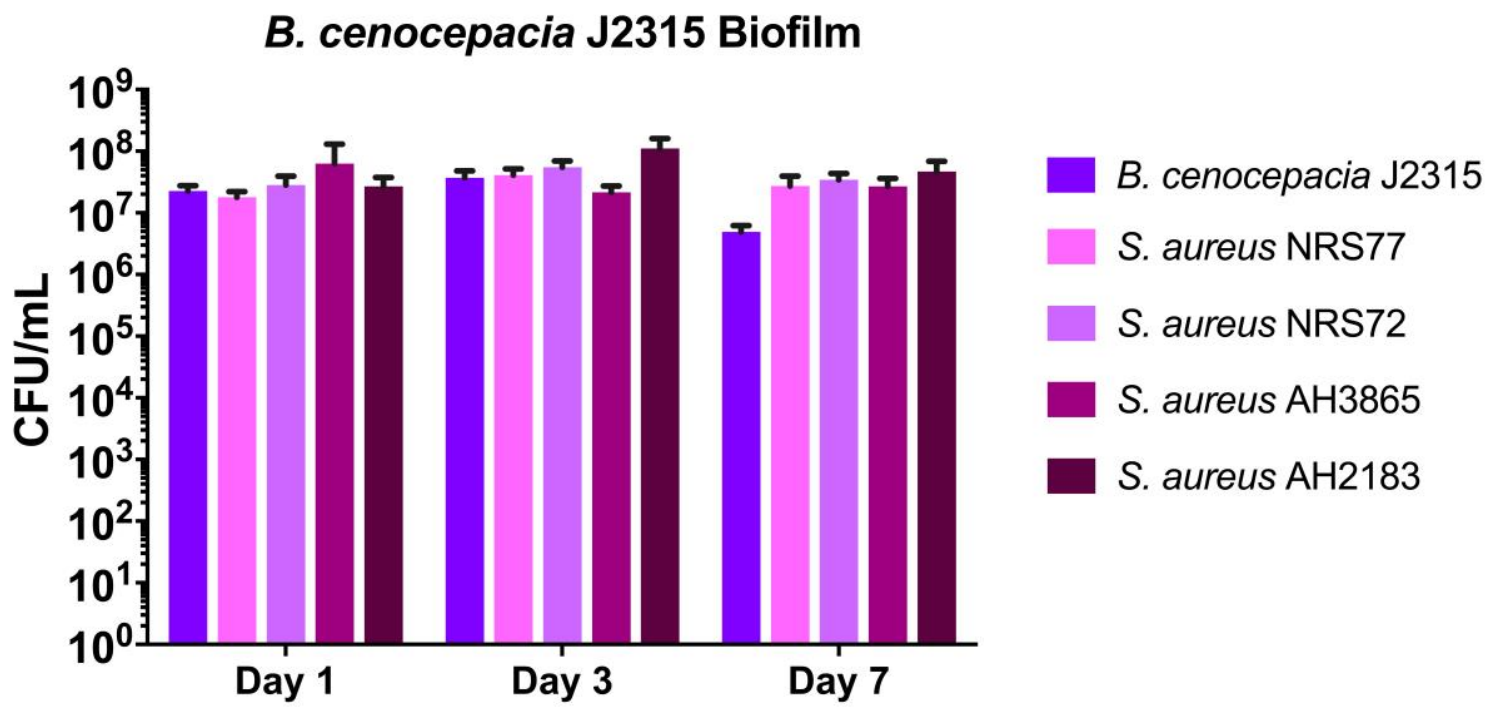

Figure 3. Effect of $S$. aureus strains on $B$. cenocepacia $\mathbf{J} 2315$ survival in co-culture biofilms. B. cenocepacia J2315 was mixed with $S$. aureus strains NRS77, NRS72, AH3862, or AH2183 for 1,3 , or 7 days. Biofilm formation was quantitated using sonication and drip dilutions, and biofilm viable cell counts were compared to monoculture biofilm growth of B. cenocepacia J2315. Oneway ANOVA with Tukey's multiple comparison test showed no significant difference over time or between co-culture conditions.

S. aureus AH3865 was able to form biofilm as effectively in the presence of $B$. cenocepacia $\mathbf{J} 2315$ as in monoculture during day 1, but was significantly inhibited by day 3 (Fig. 4C). S. aureus AH2183 biofilm formation appeared to be inhibited in the presence 
of B. cenocepacia $\mathrm{J} 2315$ after 1 day, but this effect was not statistically significant (Fig. 4D). On day 3, however, the decrease in S. aureus AH2183 co-culture biofilm was statistically significant in comparison to monoculture. All strains of $S$. aureus had no detectable biofilm on day 7 in the presence of B. cenocepacia J2315. This data suggests that B. cenocepacia $\mathrm{J} 2315$ inhibits biofilm formation in multiple $S$. aureus strains.
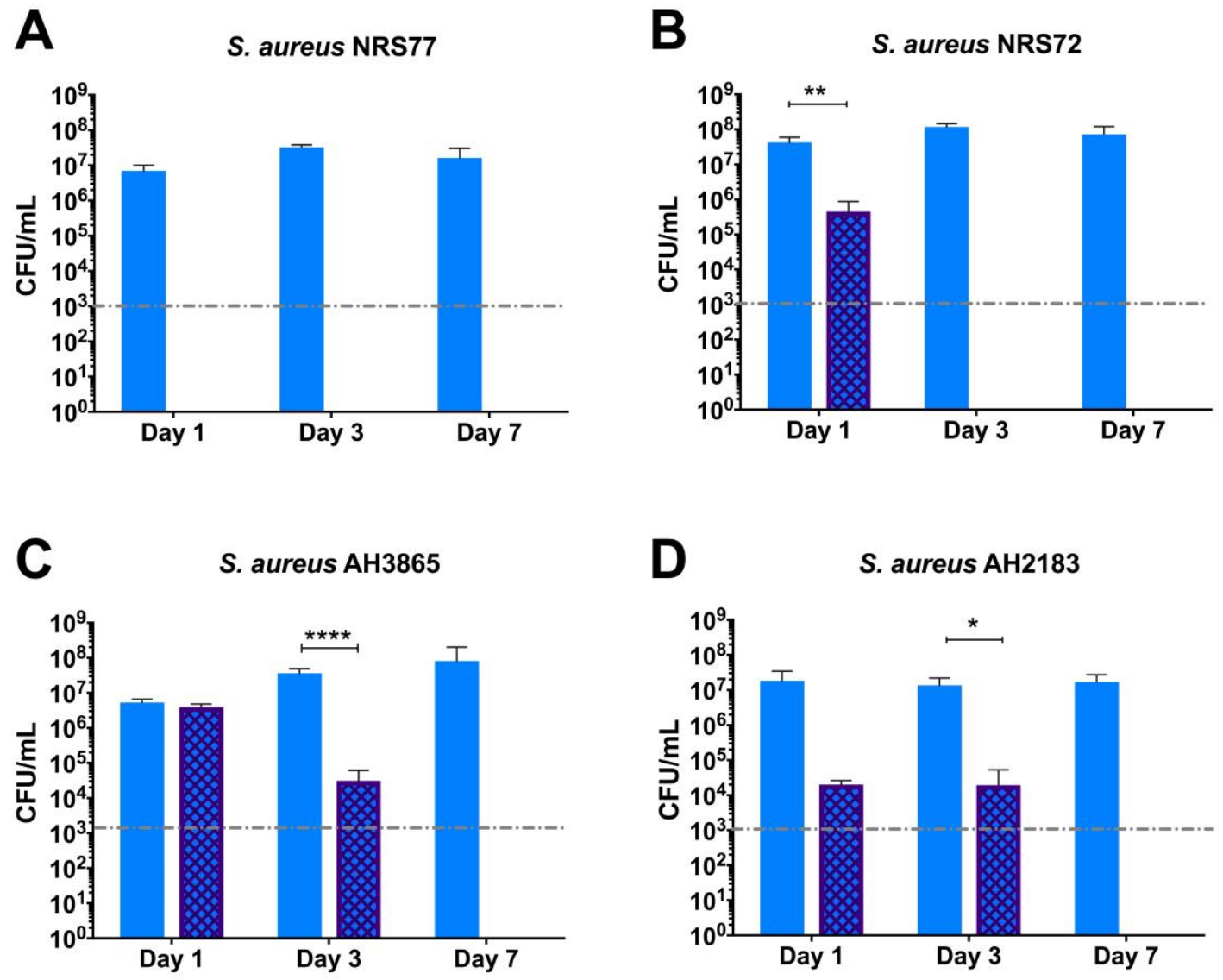

\section{S. aureus monoculture}

B. cenocepacia J2315

Figure 4. Effect of $B$. cenocepacia $\mathbf{J} 2315$ on multiple $S$. aureus strains during biofilm formation over time. S. aureus strains NRS77 (A), NRS72 (B), AH3865 (C), and AH2183 (D) were grown in monoculture and in co-culture with B. cenocepacia J2315 for 1, 3, or 7 days. Biofilm formation was quantitated using sonication and drip dilutions. Bars representing biofilm formation of $S$. aureus strains grown alone (blue) or grown in co-culture with B. cenocepacia J2315 (purple striped) are shown. Limit of detection is indicated by the gray line. Unpaired t-test measured significant differences as indicated by brackets and asterisks $(* p<0.05$, $* * p<0.01$, $* * * * \mathrm{p}<0.0001)$. 


\section{$\underline{\text { Standardizing the Viable Cell Count of Isolates with O.D. } 600}$}

In the previous experiments, an equal volume of each overnight culture was used when inoculating 96-well plates for co-culture biofilm formation. However, because $S$. aureus is a coccus and B. cenocepacia is a bacillus, the size difference between species may result in a different concentration of cells per unit volume of medium. Additionally, the viable cell count of overnight cultures within one strain may not be consistent between replicates. We sought to eliminate the difference in cell number between $B$. cenocepacia J2315 and S. aureus NRS77 as a possible source of variation.

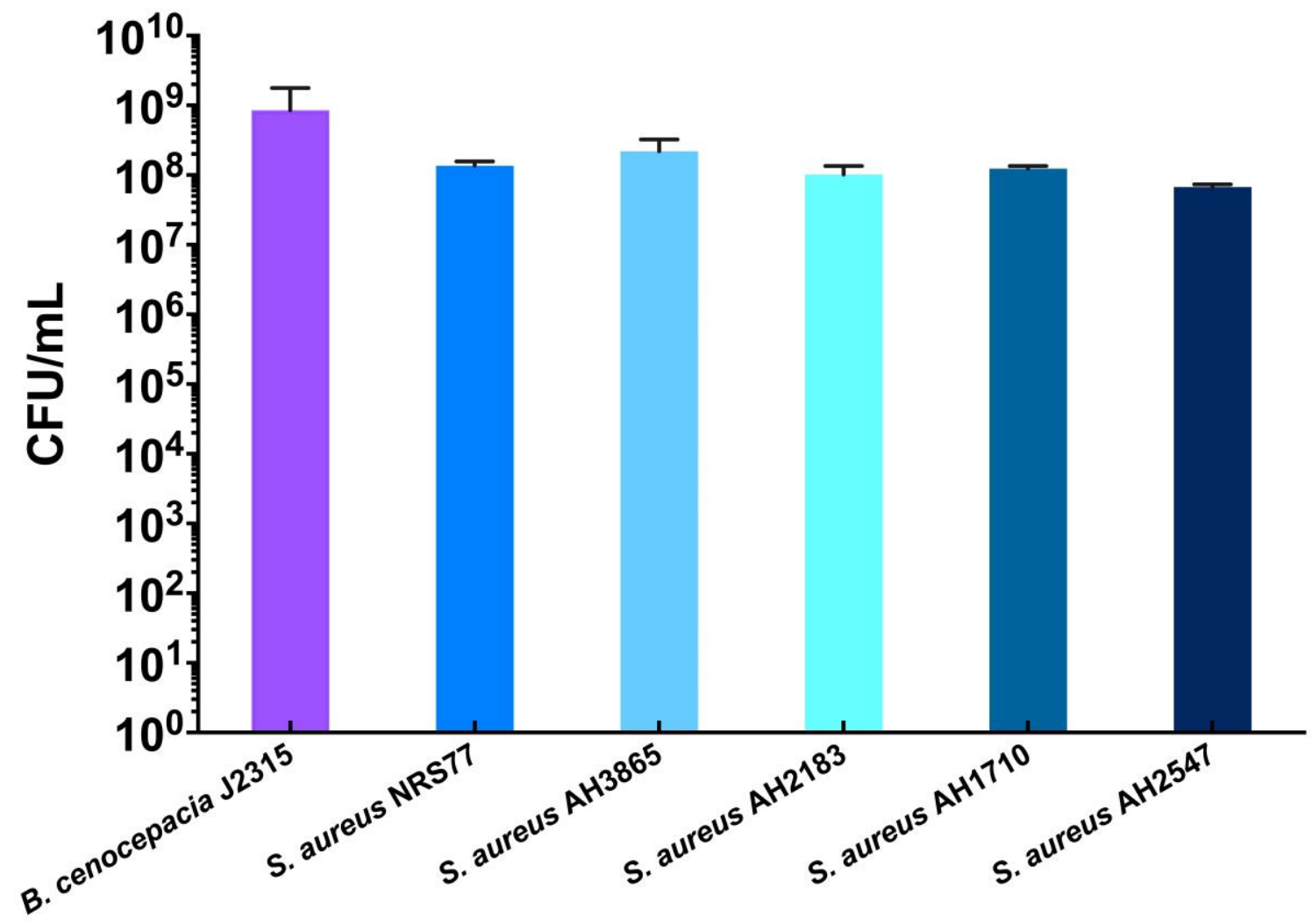

Figure 5. Viable cell count of isolates with O.D.600 of 0.8. Overnight cultures were diluted in fresh LB and grown to O.D. 600 of 0.8, serial diluted, and plated. Viable cell counts were collected for B. cenocepacia $\mathrm{J} 2315$, and the indicated $S$. aureus. One-way ANOVA analysis showed no significant differences between strains. 
Performing drip dilutions on cultures with an O.D.600 of 0.8 standardizes the viable cell count of each strain. This process was completed for B. cenocepacia J2315 and for S. aureus strains NRS77, AH3865, AH2183, AH1710, and AH2547. Results show that all S. aureus strains had an average concentration of approximately $10^{8}$ CFU/mL at this O.D.600 reading, while B. cenocepacia J2315 had an average concentration around $10^{9} \mathrm{CFU} / \mathrm{mL}$ (Fig. 5). Therefore, when creating 50/50 mixes of $B$. cenocepacia J2315 and any of the aforementioned S. aureus strains grown to O.D.600 of 0.08, B. cenocepacia $\mathbf{J} 2315$ may have 10 -fold the number of cells compared to S. aureus. This led to a fundamental change in our experimental design. In the following experiments, after strains reached an O.D.600 of 0.8, B. cenocepacia J2315 was diluted 10-fold in comparison to $S$. aureus NRS77 to account for differences in cell size.

\section{Effect of B. cenocepacia J2315 on Pre-Established S. aureus NRS77 Biofilms}

The results from the above experiments show that the J2315 strain of $B$. cenocepacia is able to prevent $S$. aureus from forming and maintaining the biofilm lifestyle. We sought to determine if B. cenocepacia $\mathbf{J} 2315$ is also able to destroy mature S. aureus NRS77 biofilms. We hypothesized that mature S. aureus biofilms would be able to survive inoculation of B. cenocepacia $\mathbf{J} 2315$, but biofilm viable cell counts would show a progressive decrease over time, while B. cenocepacia J2315 biofilms would be unaffected.

S. aureus biofilms were formed for 3 days before the addition of either more $S$. aureus NRS77, a 50/50 mixture of S. aureus NRS77 and B. cenocepacia J2315, or B. cenocepacia J2315. After 1 day, there was no significant difference between S. aureus 
biofilms of any treatment (Fig. 6). On day 3, there was a significant decrease in S. aureus NRS77 biofilm in both the treatment with B. cenocepacia J2315 / S. aureus NRS77 mixture and the treatment with $B$. cenocepacia J2315. Only the treatment with $B$. cenocepacia J2315 showed a statistically significant decrease in S. aureus NRS77 biofilm formation on day 7. In addition, compared to monoculture conditions, there was no statistically significant difference in B. cenocepacia J2315 biofilm formation in the presence of S. aureus NRS77 biofilms (data not shown).

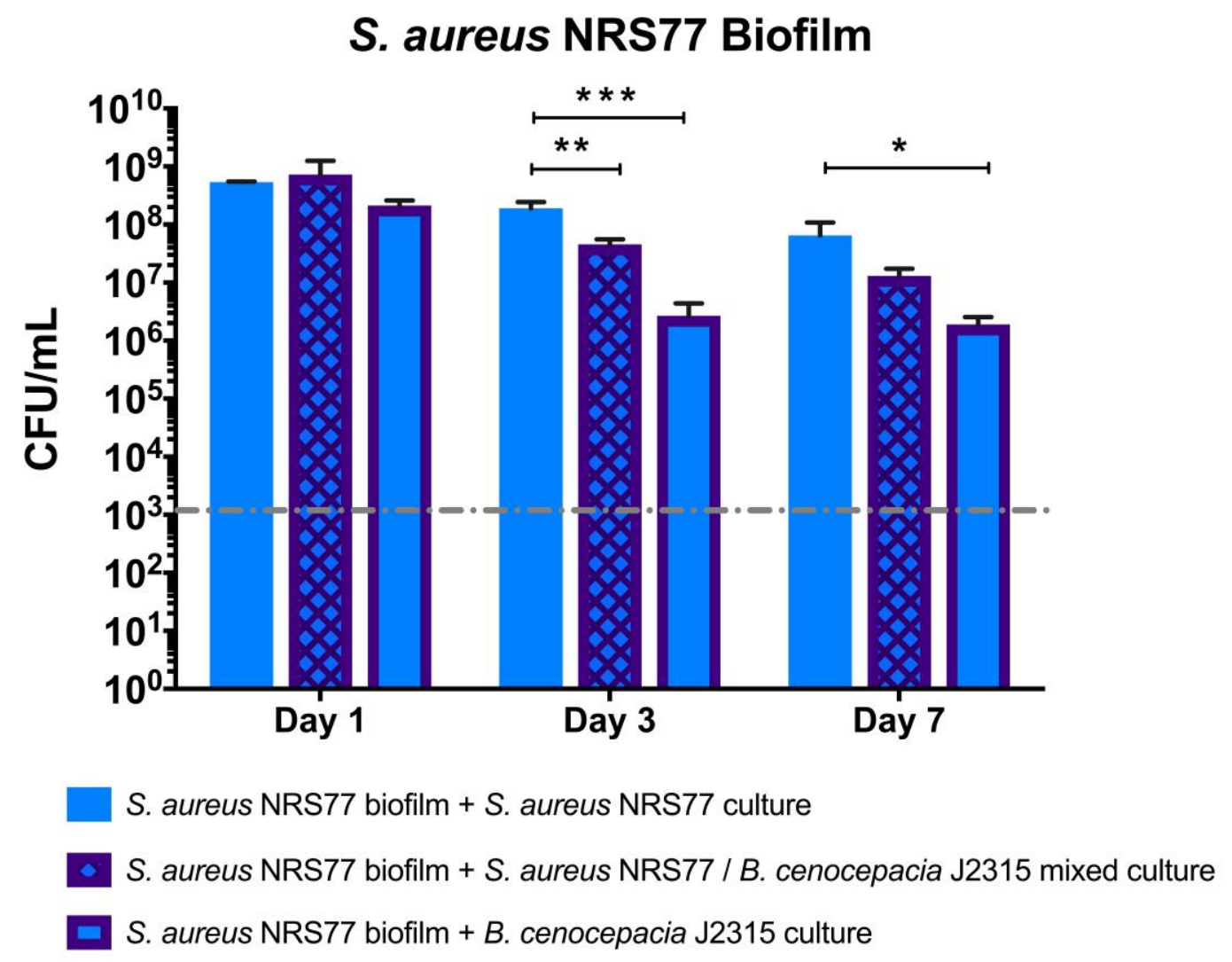

Figure 6. Inhibition of mature $S$. aureus NRS77 biofilms by B. cenocepacia J2315. Mature, 3day old $S$. aureus NRS77 biofilms were inoculated with $S$. aureus NRS77 (blue), a 50:50 mixture of S. aureus NRS77 and B. cenocepacia J2315 cultures (purple striped), or B. cenocepacia J2315 (blue bar, purple outline) and grown for 1, 3, and 7 days. Biofilms were quantitated for each condition using sonication and drip dilutions. Limit of detection is indicated by a gray line. Oneway ANOVA with Tukey's multiple comparison tests analyzed significant differences of biofilm formation between treatments $(* \mathrm{p}<0.05, * * \mathrm{p}<0.01 * * * \mathrm{p}<0.001)$. 
Together, these data continue to support the hypothesis that B. cenocepacia J2315 is diminishing the ability of $S$. aureus NRS77 to form or maintain a biofilm state. Not only does B. cenocepacia J2315 prevent biofilm formation of S. aureus NRS77 upon simultaneous inoculation, but it also results in the reduction of $S$. aureus NRS77 biofilm that has already been established. It is not clear if this inhibition is due to the presence of planktonic B. cenocepacia J2315, B. cenocepacia J2315 biofilm, or a combination of both.

\section{Exploring the Mechanism of S. aureus Biofilm Inhibition}

This experiment was designed to determine if inhibition of $S$. aureus biofilm by B. cenocepacia was through a secreted or shed product, and to determine if live $B$. cenocepacia cells are necessary for $S$. aureus biofilm destruction. Five B. cenocepacia J2315 treatments for S. aureus biofilms were chosen to answer these mechanistic questions and are depicted in Table 2. B. cenocepacia $\mathbf{J} 2315$ seems to specifically inhibit biofilm formation in S. aureus and does not appear to reduce biofilm of other tested species (Supplementary Fig. 1). Due to these observations, it was hypothesized that $B$. cenocepacia J2315 needed to recognize the presence of S. aureus NRS77 to mediate biofilm reduction, and would, therefore, require the presence of live B cenocepacia J2315 cells.

For clarification, the results from this experiment have been separated into four sections: the effect of sonicated B. cenocepacia J2315 cells, B. cenocepacia J2315 biofilm supernatant, B. cenocepacia J2315 overnight supernatant, and B. cenocepacia J2315 pelleted planktonic cells on $S$. aureus NRS77 biofilms. The effects of all $B$. 
cenocepacia J2315 fractions on S. aureus NRS77 biofilms were compared to monoculture S. aureus NRS77 biofilm formation. In addition, results could be compared to the inhibition of $S$. aureus NRS77 biofilms treated with whole B. cenocepacia J2315 culture. This was the same condition tested in previous experiments and therefore acted as a positive control for $S$. aureus NRS77 biofilm inhibition.

Table 2. Experimental design for exploring possible mechanisms of $\boldsymbol{B}$. cenocepacia J2315-mediated destruction of $S$. aureus NRS77 biofilm

\begin{tabular}{|c|c|c|}
\hline Treatment & Source & Purpose or question addressed \\
\hline $\begin{array}{l}\text { Whole B. cenocepacia } \\
\text { J2315 }\end{array}$ & Overnight culture & $\begin{array}{l}\text { Positive control for } S \text {. aureus NRS77 } \\
\text { biofilm inhibition }\end{array}$ \\
\hline $\begin{array}{c}\text { Sonicated } B . \\
\text { cenocepacia } \mathrm{J} 2315\end{array}$ & $\begin{array}{l}\text { Overnight culture, } \\
\text { sonicated }\end{array}$ & $\begin{array}{l}\text { (1) Is live } B \text {. cenocepacia } \mathbf{J} 2315 \text { necessary } \\
\text { for } S \text {. aureus biofilm reduction? }\end{array}$ \\
\hline $\begin{array}{l}\text { B. cenocepacia } \mathrm{J} 2315 \\
\text { biofilm supernatant }\end{array}$ & $\begin{array}{c}\text { Filter-sterilized } \\
\text { supernatant of a 3-day-old } \\
\text { monoculture biofilm }\end{array}$ & $\begin{array}{l}\text { (1) Do biofilm secretions result in } S \text {. } \\
\text { aureus biofilm reduction? } \\
\text { (2) Is cell-to-cell contact necessary for } S \text {. } \\
\text { aureus biofilm reduction? }\end{array}$ \\
\hline $\begin{array}{l}\text { B. cenocepacia } \mathrm{J} 2315 \\
\text { overnight supernatant }\end{array}$ & $\begin{array}{l}\text { Filter-sterilized } \\
\text { supernatant of an } \\
\text { overnight culture }\end{array}$ & $\begin{array}{l}\text { (1) Do planktonic secretions result in } S \text {. } \\
\text { aureus biofilm reduction? } \\
\text { (2) Is cell-to-cell contact necessary for } S \text {. } \\
\text { aureus biofilm reduction? }\end{array}$ \\
\hline $\begin{array}{l}\text { Pelleted/resuspended } \\
\text { B. cenocepacia }\end{array}$ & $\begin{array}{l}\text { Overnight culture, pelleted } \\
\text { and resuspended in fresh } \\
\text { medium }\end{array}$ & $\begin{array}{l}\text { (1) Are cells alone sufficient to induce } S \text {. } \\
\text { aureus biofilm reduction? } \\
\text { (2) Is cell-to-cell contact necessary for } S \text {. } \\
\text { aureus biofilm reduction? }\end{array}$ \\
\hline
\end{tabular}

The effect of sonicated B. cenocepacia J2315 cells on S. aureus NRS77 biofilms

B. cenocepacia J2315 was subjected to sonication in order to lyse cells. Following the sonication treatment, serial and drip dilutions of lysed cells suggested no detectable levels of viable B. cenocepacia cells remained (data not shown). This sonicated mixture was mixed with TSB 1:1 (v/v) and added to one-day-old S. aureus NRS77 biofilm. Biofilms were incubated for 3 days. The resulting data shows that there 
was no significant difference in the viable cell count of the $S$. aureus NRS77 biofilm and the $S$. aureus biofilm exposed to sonicated B. cenocepacia J2315 (Fig. 7). In comparison, the significant inhibition of the $S$. aureus NRS77 biofilm due to the B. cenocepacia J2315 control on day three suggests that the process that leads to S. aureus biofilm antagonism requires live $B$. cenocepacia $\mathrm{J} 2315$.

\section{S. aureus NRS77 Biofilm}

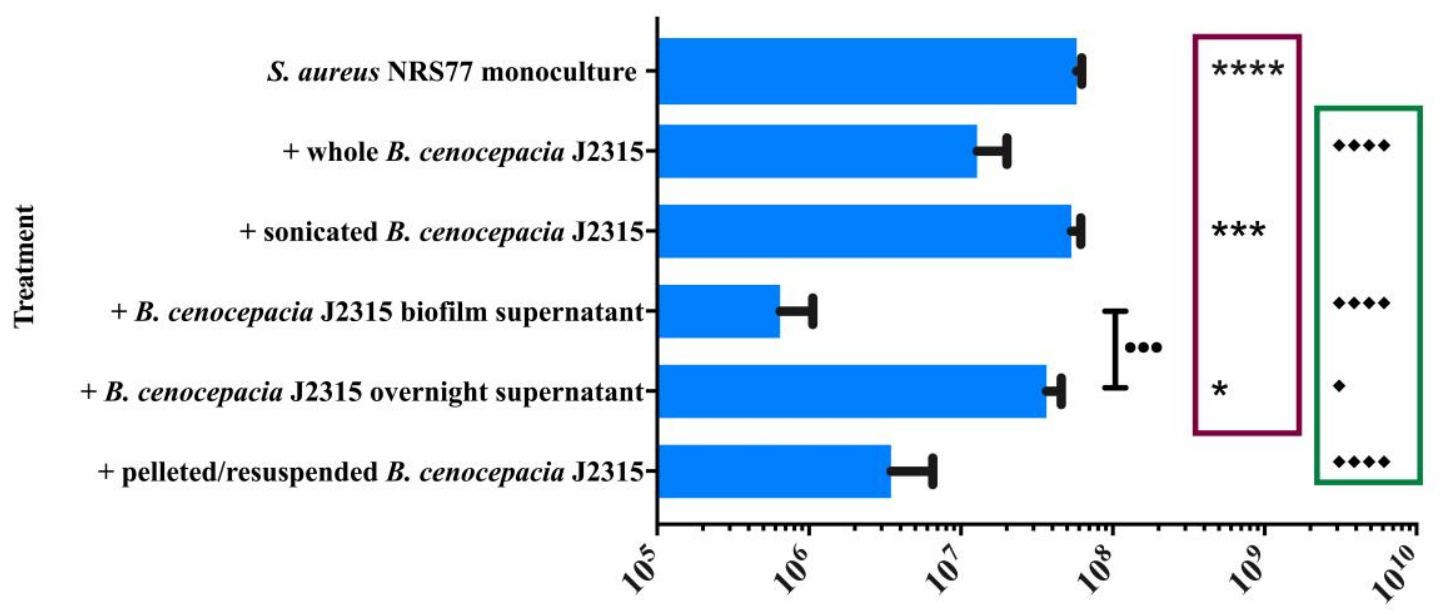

$\mathrm{CFU} / \mathrm{mL}$

Figure 7. Effect of B. cenocepacia $\mathbf{J 2 3 1 5}$ components on $\boldsymbol{S}$. aureus NRS77 biofilm. One-day-old $S$. aureus NRS77 biofilm was inoculated with either TSB or one of five experimental treatments. $S$. aureus NRS77 biofilm was quantitated after three days using sonication and drip dilutions. One-way ANOVA analysis showed a significant effect of treatment $(p<0.0001)$, and Tukey's multiple comparison test analyzed for significant difference of treatments in comparison to the treatment with whole B. cenocepacia $\mathrm{J} 2315$ (Red box; *p $<0.05$, ***p $<0.001, * * * * \mathrm{p}<0.0001$ ) and the $S$. aureus monoculture condition (Green box; $\diamond \mathrm{p}<0.05$, $\bullet \bullet \bullet p<0.0001$ ). Statistical difference of $S$. aureus NRS77 biofilm formation between B. cenocepacia J2315 overnight and biofilm supernatant treatments is also shown $(\bullet \bullet p<0.001)$.

The effect of $\mathrm{B}$. cenocepacia J2315 biofilm supernatant on $\mathrm{S}$. aureus NRS77 biofilms

B. cenocepacia J2315 biofilm was grown for three days, and the supernatant was collected and filter-sterilized before being mixed with TSB for addition to a one-day-old S. aureus NRS77 biofilm. After incubation for 3 days, the S. aureus NRS77 biofilm viable cell count was measured following sonication and drip dilutions. Analysis of the 
resulting data shows exposure to the biofilm supernatant caused a statistically significant decrease in S. aureus NRS77 biofilms compared to the S. aureus NRS77 monoculture biofilms. There was no statistically significant difference in S. aureus NRS77 biofilm inhibition when comparing the exposure to whole B. cenocepacia $\mathrm{J} 2315$ to the exposure to B. cenocepacia $\mathrm{J} 2315$ biofilm supernatant.

Both the presence of $B$. cenocepacia $\mathrm{J} 2315$ cells and the exposure to $B$. cenocepacia $\mathbf{J} 2315$ biofilm supernatant results in a reduction of S. aureus NRS77 biofilm. This suggests that B. cenocepacia $\mathrm{J} 2315$ is secreting or shedding a substance during biofilm formation that is a potential inhibitor of S. aureus NRS77 biofilms. Additionally, B. cenocepacia $\mathrm{J} 2315$ does not require the presence of $S$. aureus NRS77 for production of the inhibiting substance.

The effect of $\mathrm{B}$. cenocepacia J2315 overnight supernatant on $\mathrm{S}$. aureus NRS77 biofilms

The overnight culture of B. cenocepacia J2315 was centrifuged and the supernatant was collected, filter-sterilized, and stored in a 1:1 mixture of a glycerol solution for storage until use. On the day of inoculation of one-day-old S. aureus NRS77 biofilm, the supernatant glycerol solution was mixed 1:1 (v/v) with TSB and added to $S$. aureus NRS77 biofilms. Quantitative analysis through sonication and drip dilutions was completed for S. aureus NRS77 biofilms after three days of incubation.

B. cenocepacia J2315 overnight supernatant decreased S. aureus NRS77 biofilm to a mild but significantly lower level compared to treatment with whole $B$. cenocepacia J2315 (Fig. 7). These results suggest that B. cenocepacia J2315 may be able to secrete or shed a product during growth of overnight culture that results in a slight reduction of $S$. 
aureus NRS77 biofilms. However, because the effect of the overnight supernatant treatment did not result in the same level of inhibition as whole cultures, this suggests that either an inhibitory molecule is not optimally expressed during overnight growth or that there is an additive effect of B. cenocepacia J2315 cells and supernatant on S. aureus biofilm inhibition.

The effect of pelleted planktonic cells on S. aureus NRS77 biofilms

An overnight culture of B. cenocepacia J2315 was centrifuged, and the supernatant was removed. The resulting pellet was resuspended and diluted in fresh TSB then added to one-day-old S. aureus NRS77 biofilm and incubated for one or three days. Biofilm was quantitated through sonication and drip dilutions. Statistical analysis showed that after three days the viable cell count of $S$. aureus NRS77 biofilms was statistically lower than the monoculture $S$. aureus NRS77 biofilms but not statistically different than the S. aureus NRS77 biofilms exposed to whole B. cenocepacia J2315 (Fig. 7). This result indicates that $B$. cenocepacia $\mathbf{J} 2315$ cells, both with and without supernatant from overnight growth, are capable of reducing $S$. aureus NRS77 biofilm to the same extent.

Altogether, the data presented above suggest that our original hypothesis regarding a potential mechanism of $S$. aureus biofilm reduction was likely incorrect. $B$. cenocepacia J2315 does not require the presence of S. aureus NRS77 in order to produce an inhibitory effect on S. aureus NRS77 biofilm viability. It appears as if there is an antagonistic substance in the supernatant of B. cenocepacia $\mathbf{J} 2315$ - both from cells in a planktonic and biofilm lifestyle - that interacts with S. aureus NRS77 biofilm in a way that leads to a reduction in the viable cell count of cells in the biofilm. 


\section{Transformation of B. cenocepacia $\mathrm{J} 2315$}

To better understand the interactions between $S$. aureus strains and $B$. cenocepacia J2315 in biofilms, strains encoding fluorescent proteins were obtained. $S$. aureus strains bearing GFP- and dsRedExpress-encoding plasmids (Table 1) were obtained from Dr. Alexander Horswill at the University of Iowa. To generate fluorescent B. cenocepacia J2315, a protocol established by Dubarry et al. [116] was followed, and transformants of B. cenocepacia J2315 expressing GFP (J2315-301), mCherry (not named) and dsRedExpress (J2315-62) were created. The mCherry transformants showed weak fluorescence and were quick to photobleach, while both the GFP strain and dsRedExpress strains showed strong fluorescence that did not fade quickly. The latter two strains were used in conjunction with the provided S. aureus strains to observe coculture biofilms with confocal laser scanning microscopy, which allows 3-dimensional biofilm structures to be observed.

\section{Phenotypes of Co-culture Biofilms}

To observe the phenotypic and spatial interaction between these organisms, strains B. cenocepacia J2315-62 (red fluorescence) and S. aureus AH1710 (green fluorescence) were grown in triplicate in both co-culture and monoculture conditions for 1,3 , or 7 days in glass-bottomed petri dishes. In addition, another set of dishes were inoculated with S. aureus AH1710 monoculture and incubated for 3 days. The S. aureus supernatant was then replaced with filter-sterilized supernatant of 3-day-old $B$. cenocepacia J2315 biofilm, and the dishes were incubated with the remaining plates. It was hypothesized that the destruction of $S$. aureus biofilm formed in co-culture with $B$. 
cenocepacia J2315-62 would be observable over time. Additionally, because $B$. cenocepacia $\mathrm{J} 2315$ reduces biofilm in several $S$. aureus strains, we also predicted that $S$. aureus AH1710 biofilm exposed to B. cenocepacia J2315 biofilm supernatant would be reduced.

First, S. aureus AH1710 and B. cenocepacia J2315-62 form morphologically different biofilms in monoculture compared to co-culture. Single cultures of S. aureus AH1710 laid down a biofilm in a flat mat, with little to no clustering morphology (Figs. 8A and B), while S. aureus AH1710 cells in co-cultures with B. cenocepacia can regularly be seen in both flat mats on the surface of the dish and in mixed species mushroom structures with B. cenocepacia J2315-62 (Figs. 8E and F). The biofilm formed by $B$. cenocepacia $\mathbf{J} 2315-62$ in monoculture is highly clustered and porous, resembling honeycomb (Figs. 8C and D). These structures are less apparent when grown in coculture with $S$. aureus AH1710, where as B. cenocepacia biofilm phenotype appears to switch from a porous morphology to a mushroom-like structure that surround clusters of S. aureus (Figs. 8E and F).

While co-culture experiments using 96-well PVC plates showed an absence of biofilm of four $S$. aureus strains by day 7 when inoculated simultaneously with $B$. cenocepacia J2315 (Figs. 1 and 2), S. aureus AH1710 is still present in the co-culture biofilm after the same amount of time in co-culture, albeit to a reduced degree (Fig. 9B). However, S. aureus AH1710 biofilm exposed to 3-day-old sterile supernatant of $B$. cenocepacia $\mathrm{J} 2315$ appears markedly decreased in comparison to the co-culture condition. This difference in $S$. aureus biofilm reduction can be seen in Figure 9B and C. 
Day 3
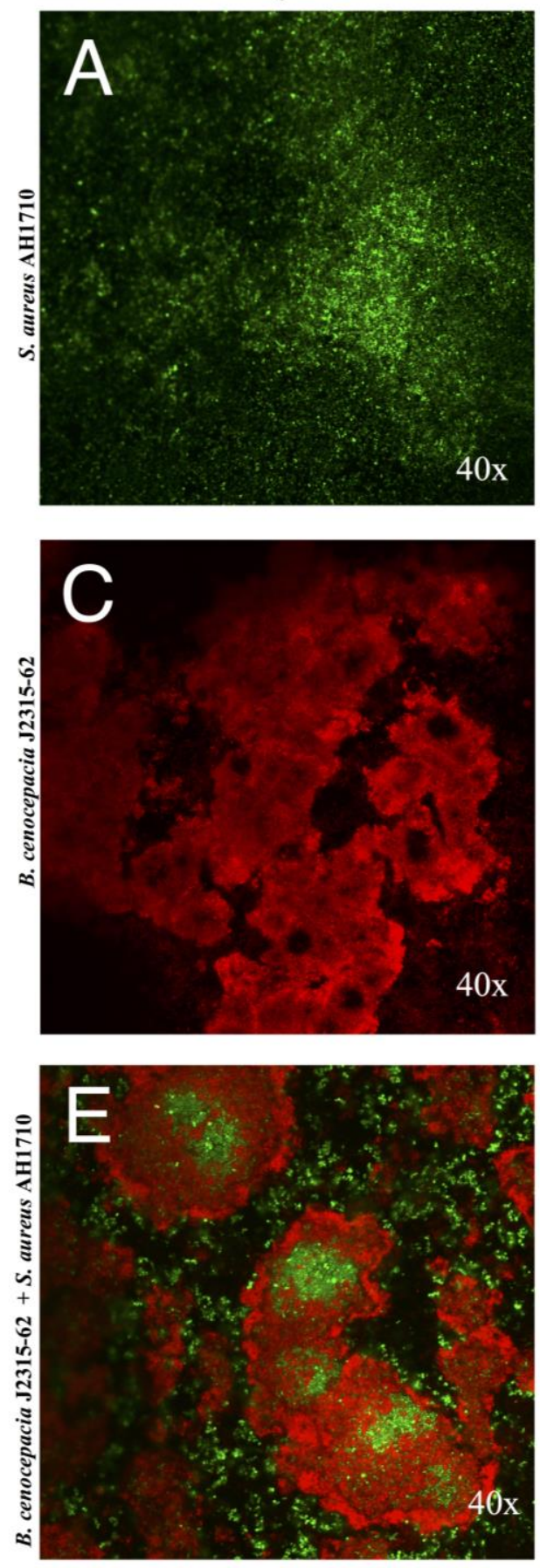

Day 7
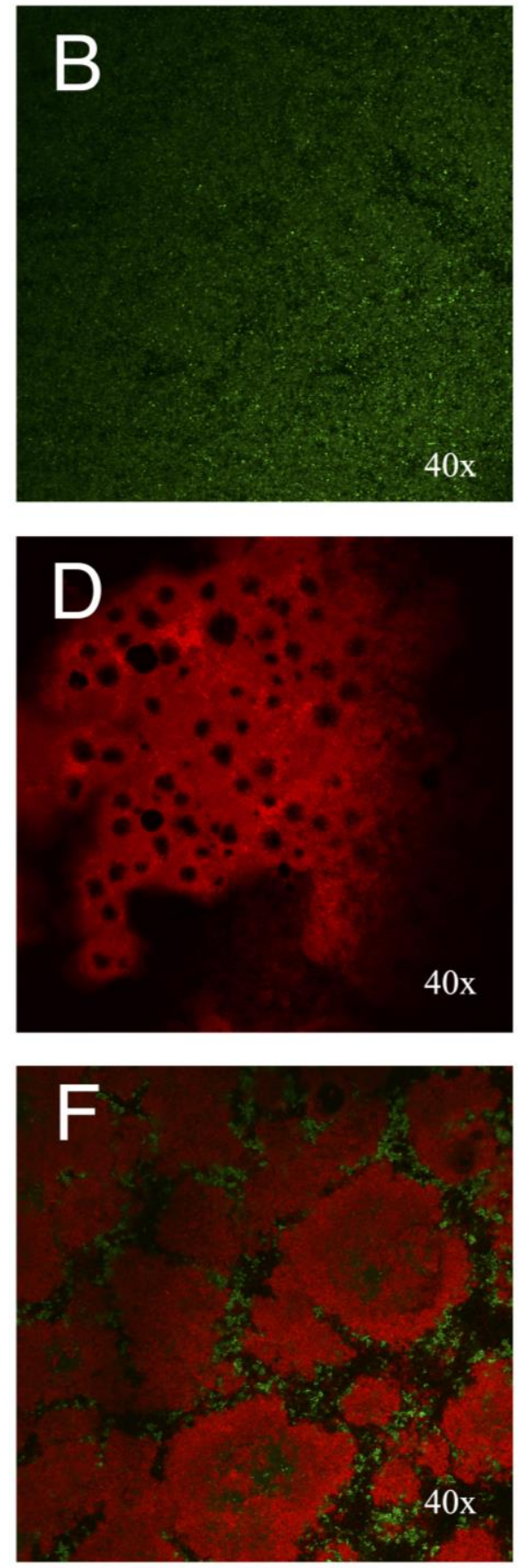

Figure 8. CLSM imaging of single and co-culture biofilms of $B$. cenocepacia $\mathrm{J} 2315-62$ and $S$. aureus AH1710 over time. Triplicate glass bottom dishes were inoculated with either single cultures (A-D) or co-cultures (E and F) of B. cenocepacia J2315-62 and S. aureus AH1710 and grown for 3 (A, C, and E) and 7 days (B,D, and F). Images were captured using NIS-Elements software, and settings were optimized for each image. 
Based on these observations, it seems as if the presence of B. cenocepacia cells may be slowing the progression of $S$. aureus biofilm destruction that results from $B$. cenocepacia J2315 secretions when grown in the glass bottom dishes. Alternatively, the timing of biofilm inhibition may be variable between cultures grown in PVC 96-well plates and cultures grown in glass-bottom dishes. A longer time period may be required to see the same levels of inhibition in the glass bottom dishes that is observed in the 96well PVC plate.
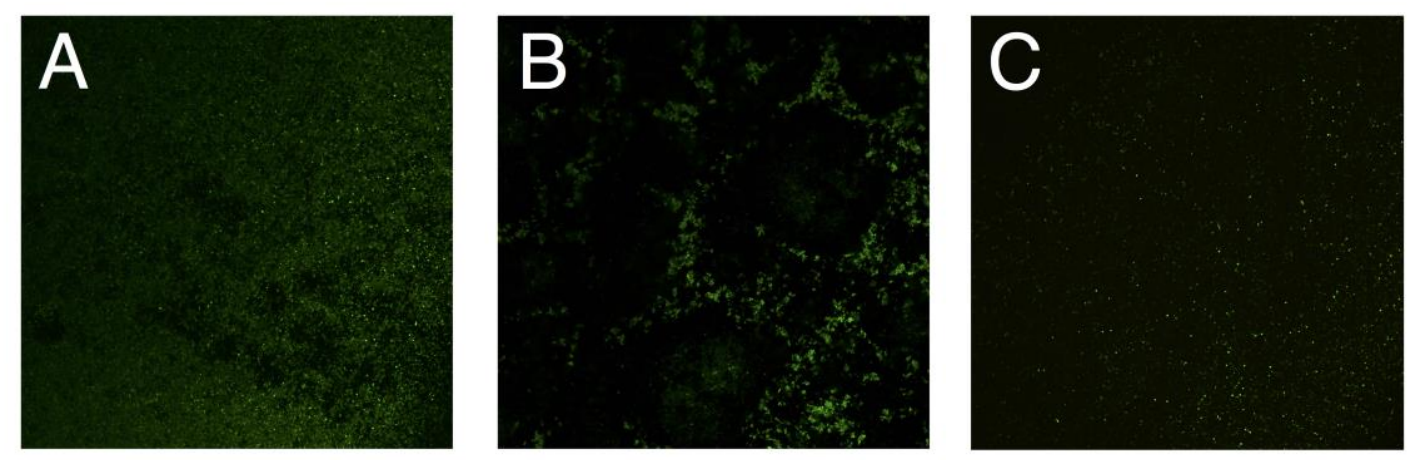

Figure 9. Effect of B. cenocepacia J2315 biofilm supernatant on $S$. aureus AH1710 biofilm. (A) Monoculture, 7-day-old S. aureus AH1710 biofilm. (B) A 7-day-old co-culture biofilm image with the red channel (B. cenocepacia J2315-62) removed, and only the green channel (S. aureus AH1710) displayed. (C) Three-day-old S. aureus AH1710 biofilm inoculated with $25 \% \mathrm{~B}$. cenocepacia $\mathbf{J} 2315$ biofilm supernatant in TSB, and incubated for an additional four days.

Although it was shown that both B. cenocepacia J2315 cells and biofilm supernatant reduce $S$. aureus biofilm to the same extent (Fig. 7), it is clear that some sort of alternate interaction is occurring under the conditions of this experiment. The presence of B. cenocepacia cells still reduces $S$. aureus biofilm, but appears to slow that reduction compared to the presence of biofilm supernatant alone. This effect could be due to the difference in the experimental design between the 96-well plate experiments and the CLSM experiments. The glass-bottom dishes used for biofilm attachment in this experiment held a larger volume of inoculated medium compared to the 96-well PVC 
plates used in other experiments. Difference in material or total medium volume may influence the time it takes for reduction in S. aureus biofilm to occur.

Another possibility is that the substance in the biofilm secretion that results in $S$. aureus biofilm destruction is regulated and taken back up by B. cenocepacia. The presence of B. cenocepacia J2315-62 would decrease the concentration of the biofilmdestroying-substance, thereby slowing $S$. aureus biofilm degradation in a dose-dependent manner. This is speculative, and further experimentation needs to be done to identify the source of S. aureus biofilm-degradation in the B. cenocepacia $\mathrm{J} 2315$ biofilm supernatant.

\section{Discussion}

Most of the published literature chronicling CF pathogenesis focus on single culture biofilms of pathogens either in vitro or in vivo; however, co-culture biofilms are common in nature and in human hosts during the course of disease and thus warrant further study. Multiple strains and species are commonly cultured from CF lungs [103], and the unique interactions that may occur in the biofilms of these isolates could provide key insight into the varying pathogenicity of the disease. The analysis of co-culture biofilm formation of B. cenocepacia and S. aureus explored in this study has shown a novel interaction with important implications.

In this study, we found that B. cenocepacia J2315 secretions result in the degradation of $S$. aureus biofilm and prevent $S$. aureus biofilm formation in several strains. Even when given the opportunity to form mature biofilms, B. cenocepacia J2315 
degrades S. aureus biofilm over time. In contrast, the presence of S. aureus NRS77 does not seem to alter biofilm formation or maintenance in B. cenocepacia $\mathbf{J} 2315$.

The exact mechanism of this interaction remains unclear, although there exist several explanative possibilities. First, B. cenocepacia secretion may be altering the $\mathrm{pH}$ of the medium. However, destruction of S. aureus biofilm is not likely occurring due to decreasing the $\mathrm{pH}$. The $\mathrm{CF}$ lung, which $S$. aureus frequently infects, is acidic in comparison to a healthy lung due to the improper transport of bicarbonate ions [117]. Additionally, low $\mathrm{pH}$ can be an environmental signal to initiate biofilm formation in staphylococcal species [118]. Its ability to survive in low $\mathrm{pH}$ conditions suggests a different mechanism is causing degradation of S. aureus biofilm. Further, our data suggests this inhibitory effect may be specific to $S$. aureus, as similar inhibition was not observed for P. aeruginosa or S. maltophilia (Supplementary Fig. 1). A generalized mechanism such as decreased $\mathrm{pH}$ would theoretically affect biofilm formation in other species as well. Absence of this effect insinuates that the substance secreted by $B$. cenocepacia J2315 specifically targets S. aureus or a component of the $S$. aureus biofilm.

Another possible mechanism leading to $S$. aureus biofilm destruction is through the destruction of the EPS holding the biofilm together, such as through secretions of polysaccharide hydrolases, DNases, and/or proteases. This destruction would result in release of cells from the bacterial matrix and back to planktonic growth. This explanation is easily testable by removing the liquid from a co-culture biofilm, quantitating planktonic bacteria, and comparing the viable cell count to the $\mathrm{CFU} / \mathrm{mL}$ of the planktonic culture of S. aureus monoculture biofilm. The presence of a greater viable cell count of planktonic $S$. aureus in co-culture with B. cenocepacia compared to $S$. aureus in single 
culture would suggest the biofilm matrix is being dissolved and releasing the cells back into the medium.

Destruction of $S$. aureus biofilm in such a manner would not be a novel finding. A serine protease secreted by Staphylococcus epidermidis termed Esp has been shown to degrade both molecules in the matrix of $S$. aureus biofilm and molecules essential for biofilm formation and colonization, such as fibronectin binding proteins [119]. Exposure to Esp results in degradation of established $S$. aureus biofilm over time in a similar manner as exposure to B. cenocepacia and its biofilm supernatant.

Other potential mechanisms to explain the antagonism of secreted compounds to S. aureus biofilm could be the production of reactive oxygen species (ROS) in the medium or the production of biofilm-inhibiting lipids. ROS secretion by B. cenocepacia is less likely, as some Bcc members are killed by certain antibiotics through a resulting increase in ROS production [120].

Production of biofilm-inhibiting lipids, on the other hand, is a possible mechanism of $S$. aureus biofilm reduction. Rhamnolipids produced by $P$. aeruginosa have anti-staphylococcal activity [121] and act to decrease biofilm formation in order to maintain water channels [122]. B. cenocepacia J2315 has been shown to possess the operon required for rhamnolipid production [123], although the production of rhamnolipids has not yet been reported in this species. However, the porous biofilm phenotype shown in our study suggests the importance of water channels in $B$. cenocepacia biofilm maturation and maintenance, hinting at a possible role or expression of the rhamnolipid regulating operon. As a typically aerobic species, access to oxygen is critical for B. cenocepacia survival. Maintenance of biofilm water channels for access to 
fresh nutrients and oxygen implicate the potential importance of rhamnolipids in $B$. cenocepacia virulence, and is therefore a potential mechanism of $S$. aureus biofilm inhibition.

In addition to rhamnolipids, $B$. cenocepacia $\mathrm{J} 2315$ produces a lipopeptide capable of lysing human cells and inhibiting growth of both $S$. aureus and $P$. aeruginosa strains [124]. However, this lipopeptide was only produced at effective concentrations with vigorous shaking for three days. Our data suggest that the substance secreted by $B$. cenocepacia J2315 that inhibits $S$. aureus biofilm is produced under static conditions for the same amount of time. It is therefore probable that the inhibition of $S$. aureus observed in our data is not occurring through actions of this lipopetide.

Further research into the identity of the B. cenocepacia J2315 secreted substance could clarify the discrepancy seen in the significant reduction of S. aureus AH1710 biofilm exposed to B. cenocepacia supernatant during CLSM imaging. Co-culture imaging showed that S. aureus AH1710 was still present in 7-day-old co-culture biofilm with $B$. cenocepacia $\mathbf{J} 2315$, while $B$. cenocepacia supernatant in the absence of viable $B$. cenocepacia cells resulted in intensified S. aureus AH1710 biofilm reduction.

A possible mechanism explaining this $S$. aureus biofilm reduction is the secretion and re-uptake of a toxic or catabolic substance by B. cenocepacia. $P$. aeruginosa has been shown to produce siderophores that are toxic to $S$. aureus and lead to cell lysis as a way to scavenge iron [125]. Siderophores are secreted to collect iron from the environment and are then taken back up by the bacteria. A similar mechanism used by $B$. cenocepacia may explain the presence of $S$. aureus biofilm in co-culture with $B$. cenocepacia (Fig. 7E and 7F) in the CLSM experiment, whereas the presence of the $B$. 
cenocepacia biofilm supernatant without B. cenocepacia cells resulted in $S$. aureus biofilm destruction (Fig. 9). If B. cenocepacia is secreting a molecule that destroys $S$. aureus biofilm but the molecule can be taken back up into the cell, then the presence of B. cenocepacia could be somewhat ameliorating to S. aureus biofilm degradation in such a situation. B. cepacia complex isolates have been shown to produce a variety of siderophores including ornibactin, pyochelin, salicylic acid, and cepabactin [126-129].

There are many hypotheses to be explored and tested as a result of the data presented in this thesis. Though the exact mechanism underlying the B. cenocepacia mediated destruction of $S$. aureus biofilm remains to be elucidated, the degradation of $S$. aureus biofilm by a potentially novel substance is an exciting finding. The increasing prevalence of antibiotic resistance in both MSSA and MRSA necessitates the discovery of both new antibacterials and targets for therapeutics.

There are antibacterials on the market that can effectively treat S. aureus infection in $\mathrm{CF}$ and other individuals [130], but antibiotic resistance by bacteria protected in biofilms prevents treatment of the underlying infection. Should further experimentation reveal that the substance secreted or shed by B. cenocepacia results in dissolution of $S$. aureus biofilm matrix, it could implicate a potential therapeutic solution to elimination of S. aureus biofilms.

Future directions for this research include the identification of the substance of interest being secreted by B. cenocepacia $\mathrm{J} 2315$, use of tissue cultures as a surface for coculture biofilm formation, analysis and comparison of gene expression between $B$. cenocepacia planktonic and biofilm lifestyles, as well as continuous capture of CLSM images to create and observe a time lapse of co-culture biofilm formation and S. aureus 
biofilm degradation. Results from these proposed experiments, used in conjunction with the findings of this paper, could reveal a novel antimicrobial agent or a new target for $S$. aureus biofilm reduction.

\section{Acknowledgments}

I would like to give my thanks and gratitude to Dr. Deborah Yoder-Himes for the never-ending support, guidance, and contagious enthusiasm she has given throughout the completion of this project. I want to thank Dr. Patricia Soucy and Betty Nunn for use of the confocal microscope and Dr. Annette Vergunst and Dr. Alexander Horswill for providing the fluorescent strains utilized in this thesis. I would also like to thank my fellow lab members, Akhila Ankem, Lauren Barrett, Kryshawna Beard, TJ Brandt, Brad Clark, Tom Hundley, and Steve Tran, for the problems they have helped me solve, the encouragement they have given, and for providing a lab environment where learning and camaraderie go hand-in-hand. Funding for this project was provided by the University of Louisville Undergraduate Research Scholar's Grant and the Biology Department. 


\section{References}

1. Palomaki, G. E. F., S. C.; Haddow, J. E. (2004). "Clinical sensitivity of prenatal screening for cystic fibrosis via CFTR carrier testing in a United States panethnic population." Genet Med. 6(5): 405-414.

2. Gustafsson, J. K. E., A; Ambort, D; Johansson, M. E.V.; Nilsson, H. E.; Thorell, K.; Hebert, H.; Sjovall, H.; Hansson, G. C. (2012). "Bicarbonate and functional CFTR channel are required for proper secretion and link cystic fibrosis with its mucus phenotype." The Journal of Experimental Medicine 209(7): 1263-1272.

3. Claustres, M., Guittard, C., Bozon, D., Chevalier, F., Verlingue, C., Ferec, C., . . . Georges, M. D. (2000). Spectrum of CFTR mutations in cystic fibrosis and in congenital absence of the vas deferens in France. Hum Mutat, 16(2), 143-156.

4. Zabner, J., Smith, J. J., Karp, P. H., Widdicombe, J. H., \& Welsh, M. J. (1998). Loss of CFTR chloride channels alters salt absorption by cystic fibrosis airway epithelia in vitro. Mol Cell, 2(3), 397-403.

5. Smith, J. J., Travis, S. M., Greenberg, E. P., \& Welsh, M. J. (1996). Cystic fibrosis airway epithelia fail to kill bacteria because of abnormal airway surface fluid. Cell, 85(2), 229-236.

6. Abou Alaiwa, M. H. R., L. R.; Gansemer, N. D.; Sheets, K. A.; Horswill, A. R.; Stoltz, D. A.; Zabner, J.; Welsh, M. J. (2014). "pH modulates the activity and synergism of the airway surface liquid antimicrobials $\beta$-defensin-3 and LL-37." Proc Natl Acad Sci U S A 111(52): 18703-18708.

7. Pezzulo, A. A.; Tang, X. X.; Hoegger, M. J.; Abou Alaiwa, M. H.; Ramachandran, S.; Moninger, T. O.; Karp, P. H.; Wohlford-Lenane, C. L.; Haagsman, H. P.; van Eijk, M.; Banfi, B.; Horswill, A. R.; Stoltz, D. A.; McCray, P. B., Jr.; Welsh, M. J; .Zabner, J. (2012). Reduced airway surface pH impairs bacterial killing in the porcine cystic fibrosis lung. Nature, 487(7405), 109-113. doi:10.1038/nature11130

8. Flynn, J. M., Niccum, D., Dunitz, J. M., \& Hunter, R. C. (2016). Evidence and Role for Bacterial Mucin Degradation in Cystic Fibrosis Airway Disease. PLoS Pathog, 12(8), e1005846. doi:10.1371/journal.ppat.1005846

9. Ashford, T. P., \& Porter, K. R. (1962). Cytoplasmic components in hepatic cell lysosomes. J Cell Biol, 12, 198-202.

10. Jessup, W., \& Dean, R. T. (1983). Endocytosis and intracellular protein degradation in cystic fibrosis fibroblasts. Clin Chim Acta, 129(3), 333-339. 
11. Luciani, A., Villella, V. R., Esposito, S., Brunetti-Pierri, N., Medina, D. L., Settembre, C., . . . Maiuri, L. (2011). Cystic fibrosis: a disorder with defective autophagy. Autophagy, 7(1), 104-106.

12. Mayer, M. L., Blohmke, C. J., Falsafi, R., Fjell, C. D., Madera, L., Turvey, S. E., \& Hancock, R. E. (2013). Rescue of dysfunctional autophagy attenuates hyperinflammatory responses from cystic fibrosis cells. J Immunol, 190(3), 1227-1238. doi:10.4049/jimmunol.1201404

13. Bonfield, T. L., Panuska, J. R., Konstan, M. W., Hilliard, K. A., Hilliard, J. B., Ghnaim, H., \& Berger, M. (1995). Inflammatory cytokines in cystic fibrosis lungs. Am $J$ Respir Crit Care Med, 152(6 Pt 1), 2111-2118. doi:10.1164/ajrccm.152.6.8520783

14. Kube, D., Sontich, U., Fletcher, D., \& Davis, P. B. (2001). Proinflammatory cytokine responses to $P$. aeruginosa infection in human airway epithelial cell lines. Am J Physiol Lung Cell Mol Physiol, 280(3), L493-502.

15. Tabary, O., Zahm, J. M., Hinnrasky, J., Couetil, J. P., Cornillet, P., Guenounou, M., . . . Jacquot, J. (1998). Selective up-regulation of chemokine IL-8 expression in cystic fibrosis bronchial gland cells in vivo and in vitro. Am J Pathol, 153(3), 921-930. doi:10.1016/S0002-9440(10)65633-7

16. Clunes, M. T. a. B., R. C. (2007). Cystic Fibrosis: The Mechanisms of Pathogenesis of an Inherited Lung Disorder. Drug Discov Today Dis Mech, 4(2), 63-72. doi:10.1016/j.ddmec.2007.09.001

17. Costerton, J. W., Stewart, P. S., \& Greenberg, E. P. (1999). Bacterial biofilms: a common cause of persistent infections. Science, 284(5418), 1318-1322.

18. Hoiby, N., Ciofu, O., \& Bjarnsholt, T. (2010). Pseudomonas aeruginosa biofilms in cystic fibrosis. Future Microbiol, 5(11), 1663-1674. doi:10.2217/fmb.10.125

19. Saukkonen, K., Sande, S., Cioffe, C., Wolpe, S., Sherry, B., Cerami, A., \& Tuomanen, E. (1990). The role of cytokines in the generation of inflammation and tissue damage in experimental gram-positive meningitis. $J$ Exp Med, 171(2), 439-448.

20. Soni, R., Dobbin, C. J., Milross, M. A., Young, I. H., \& Bye, P. P. (2008). Gas exchange in stable patients with moderate-to-severe lung disease from cystic fibrosis. J Cyst Fibros, 7(4), 285-291.

21. 2015 Annual Data Report, Cystic Fibrosis Foundation Patient Registry (2016). Cystic Fibrosis Foundtation. 
22. Thurlow, L. R.; Hanke, M. L., Fritz, T.; Angle, A., Aldrich, A.; Williams, S. H.; Engebretsen, I. L.; Bayles, K. W.; Horswill, A. R.; Kielian, T. (2011). Staphylococcus aureus biofilms prevent macrophage phagocytosis and attenuate inflammation in vivo. J Immunol, 186(11), 6585-6596. doi:10.4049/jimmunol.1002794

23. Harris, A., Torres-Viera, C., Venkataraman, L., DeGirolami, P., Samore, M., \& Carmeli, Y. (1999). Epidemiology and clinical outcomes of patients with multiresistant Pseudomonas aeruginosa. Clin Infect Dis, 28(5), 1128-1133. doi:10.1086/514760

24. Ogston, A. (1881). Report upon Micro-Organisms in Surgical Diseases. Br Med J, 1(1054), 369.b362-375.

25. Eriksen, N. H. R. E., F.; Rosdahl, V. T.; Jensen, K. (1995). Carriage of Staphylococcus aureus among 104 Healthy Persons during a 19-Month Period. Epidemiol Infect, 115(1).

26. Akobi, B., Aboderin, O., Sasaki, T., \& Shittu, A. (2012). Characterization of Staphylococcus aureus isolates from faecal samples of the Straw-Coloured Fruit Bat (Eidolon helvum) in Obafemi Awolowo University (OAU), Nigeria. BMC Microbiol, 12, 279. doi:10.1186/1471-2180-12-279

27. Espinosa-Gongora, C., Chrobak, D., Moodley, A., Bertelsen, M. F., \& Guardabassi, L. (2012). Occurrence and distribution of Staphylococcus aureus lineages among zoo animals. Vet Microbiol, 158(1-2), 228-231. doi:10.1016/j.vetmic.2012.01.027

28. Faires, M. C., Gehring, E., Mergl, J., \& Weese, J. S. (2009). Methicillinresistant Staphylococcus aureus in marine mammals. Emerg Infect Dis, 15(12), 2071-2072. doi:10.3201/eid1512.090220

29. Gomez, P., Gonzalez-Barrio, D., Benito, D., Garcia, J. T., Vinuela, J., Zarazaga, M., . . . Torres, C. (2014). Detection of methicillin-resistant Staphylococcus aureus (MRSA) carrying the mecC gene in wild small mammals in Spain. $J$ Antimicrob Chemother, 69(8), 2061-2064. doi:10.1093/jac/dku100

30. Lowe, B. A., Marsh, T. L., Isaacs-Cosgrove, N., Kirkwood, R. N., Kiupel, M., \& Mulks, M. H. (2011). Microbial communities in the tonsils of healthy pigs. Vet Microbiol, 147(3-4), 346-357. doi:10.1016/j.vetmic.2010.06.025

31. Morris, P. J., Johnson, W. R., Pisani, J., Bossart, G. D., Adams, J., Reif, J. S., \& Fair, P. A. (2011). Isolation of culturable microorganisms from free-ranging bottlenose dolphins (Tursiops truncatus) from the southeastern United States. Vet Microbiol, 148(2-4), 440-447. doi:10.1016/j.vetmic.2010.08.025 
32. Nowakiewicz, A., Zieba, P., Ziolkowska, G., Gnat, S., Muszynska, M., Tomczuk, K., . . . Troscianczyk, A. (2016). Free-Living Species of Carnivorous Mammals in Poland: Red Fox, Beech Marten, and Raccoon as a Potential Reservoir of Salmonella, Yersinia, Listeria spp. and Coagulase-Positive Staphylococcus. $\quad P L o S \quad$ ONE, $11(5), \quad$ e0155533. doi:10.1371/journal.pone.0155533

33. van Elk, C. E., Boelens, H. A., van Belkum, A., Foster, G., \& Kuiken, T. (2012). Indications for both host-specific and introduced genotypes of Staphylococcus aureus in marine mammals. Vet Microbiol, 156(3-4), 343-346. doi:10.1016/j.vetmic.2011.10.034

34. Kuroda, M. O., T; Uchiyama I; et al. (2001). Whole genome sequencing of meticillin-resistant Staphylococcus aureus. Lancet, 357, 1225-1240.

35. Fowler, V. G., Jr., Miro, J. M., Hoen, B., Cabell, C. H., Abrutyn, E., Rubinstein, E., . . Bayer, A. S. (2005). Staphylococcus aureus endocarditis: a consequence of medical progress. Jama, 293(24), 3012-3021. doi:10.1001/jama.293.24.3012

36. Inoue, S., Moriyama, T., Horinouchi, Y., Tachibana, T., Okada, F., Maruo, K., \& Yoshiya, S. (2013). Comparison of clinical features and outcomes of Staphylococcus aureus vertebral osteomyelitis caused by methicillin-resistant and methicillin-sensitive strains. Springerplus, 2(1), 283. doi:10.1186/21931801-2-283

37. Gillet, Y., Issartel, B., Vanhems, P., Fournet, J. C., Lina, G., Bes, M., . . . Etienne, J. (2002). Association between Staphylococcus aureus strains carrying gene for Panton-Valentine leukocidin and highly lethal necrotising pneumonia in young immunocompetent patients. Lancet, 359(9308), 753-759. doi:10.1016/s0140-6736(02)07877-7

38. Murdoch, D. R., Roberts, S. A., Fowler Jr, V. G., Jr., Shah, M. A., Taylor, S. L., Morris, A. J., \& Corey, G. R. (2001). Infection of orthopedic prostheses after Staphylococcus aureus bacteremia. Clin Infect Dis, 32(4), 647-649. doi:10.1086/318704

39. Tsukayama, D. T., Wicklund, B., \& Gustilo, R. B. (1991). Suppressive antibiotic therapy in chronic prosthetic joint infections. Orthopedics, 14(8), 841844.

40. Nielsen, J., Ladefoged, S. D., \& Kolmos, H. J. (1998). Dialysis catheter-related septicaemia--focus on Staphylococcus aureus septicaemia. Nephrol Dial Transplant, 13(11), 2847-2852.

41. Mohan, S. S., McDermott, B. P., \& Cunha, B. A. (2005). Methicillin-resistant Staphylococcus aureus prosthetic aortic valve endocarditis with paravalvular 
abscess treated with daptomycin. Heart Lung, 34(1), 69-71. doi:10.1016/j.hrtlng.2004.07.009

42. Chamis, A. L., Peterson, G. E., Cabell, C. H., Corey, G. R., Sorrentino, R. A., Greenfield, R. A., . . . Fowler, V. G., Jr. (2001). Staphylococcus aureus bacteremia in patients with permanent pacemakers or implantable cardioverterdefibrillators. Circulation, 104(9), 1029-1033.

43. Ahn, C. Y., Ko, C. Y., Wagar, E. A., Wong, R. S., \& Shaw, W. W. (1996). Microbial evaluation: 139 implants removed from symptomatic patients. Plast Reconstr Surg, 98(7), 1225-1229.

44. McCaig, L. F., McDonald, L. C., Mandal, S., \& Jernigan, D. B. (2006). Staphylococcus aureus-associated skin and soft tissue infections in ambulatory care. Emerg Infect Dis, 12(11), 1715-1723. doi:10.3201/eid1211.060190

45. Miethke, T., Gaus, H., Wahl, C., Heeg, K., \& Wagner, H. (1992). T-celldependent shock induced by a bacterial superantigen. Chem Immunol, 55, 172184.

46. Kitai, S., Shimizu, A., Kawano, J., Sato, E., Nakano, C., Uji, T., \& Kitagawa, H. (2005). Characterization of methicillin-resistant Staphylococcus aureus isolated from retail raw chicken meat in Japan. J Vet Med Sci, 67(1), 107-110.

47. Syne, S. M., Ramsubhag, A., \& Adesiyun, A. A. (2013). Microbiological hazard analysis of ready-to-eat meats processed at a food plant in Trinidad, West Indies. Infect Ecol Epidemiol, 3. doi:10.3402/iee.v3i0.20450

48. Crass, B. A., \& Bergdoll, M. S. (1986). Toxin involvement in toxic shock syndrome. J Infect Dis, 153(5), 918-926.

49. Staali, L. M., H.; Colin, D.A. (1998). The Staphylococcal Pore-forming Leukotoxins Open $\mathrm{Ca} 2+$ Channels in the Membrane of Human Polymorphonuclear Neutrophils. J Membr Biol, 162(3), 209-216. doi:10.1007/s002329900358

50. Kaneko, J., Ozawa, T., Tomita, T., \& Kamio, Y. (1997). Sequential binding of Staphylococcal gamma-hemolysin to human erythrocytes and complex formation of the hemolysin on the cell surface. Biosci Biotechnol Biochem, 61(5), 846-851. doi:10.1271/bbb.61.846

51. Sugawara, T., Yamashita, D., Kato, K., Peng, Z., Ueda, J., Kaneko, J., . . Y Yao, M. (2015). Structural basis for pore-forming mechanism of staphylococcal alpha-hemolysin. Toxicon, 108, 226-231. doi:10.1016/j.toxicon.2015.09.033 
52. Bantel, H., Sinha, B., Domschke, W., Peters, G., Schulze-Osthoff, K., \& Janicke, R. U. (2001). alpha-Toxin is a mediator of Staphylococcus aureusinduced cell death and activates caspases via the intrinsic death pathway independently of death receptor signaling. J Cell Biol, 155(4), 637-648. doi:10.1083/jcb.200105081

53. Fowler, T. W., E. R.; Joh, D.; Johansson, S.; Foster, T. J.; Hook, M. (2000). Cellular invasion by Staphylococcus aureus involves a fibronectin bridge between the bacterial fibronectin-bidning MSCRAMMs and the host cell B1 integrins. Eur J Cell Biol, 79(10), 672-679. doi:10.1078/0171-9335-00104

54. Peacock, S. J. D., N. P. J.; Thomas, M. G.; Berendt, A. R.; Foster, T. J. (2000). Clinical Isolates of Staphylococcus aureus Exhibit Diversity in fnb Genes and Adhesion to Human Fibronectin. $J$ Infect, 41(1), 23-31. doi:10.1053/jinf.2000.0657

55. Mazmanian, S. K. L., G.; Ton-That, H.; Schneewind, O. (1999). Staphylococcus aureus sortase, an enzyme that anchors surface proteins to the cell wall. Science, 285(5428), 760-763.

56. Ghasemian, A. P., S. N., Bakhshi, B.; Mirzaee, M. (2015). The Microbial Surface Components Recognizing Adhesive Matrix Molecules (MSCRAMMs) Genes among Clinical Isolates of Staphylococcus aureus from Hospitalized Children. Iran J Path, 10(4), 258-264.

57. Heilmann, C. N., S.; Sinha, B.; Herrman, M; Kehrel B. E.; Peters, G. (2004). Staphylococcus aureus fibronectin-binding protein (FnBP)-mediated adherence to platelets, and aggregation of platelets induced by FnBPA but not by FnBPB. J Infect Dis, 190(2), 321-329. doi:10.1086/421914

58. Schneewind, O., ; Model, P.; Fischetti, V. A. (1992). Sorting of protein A to the staphylococcal cell wall. Cell, 70(2), 267-281.

59. Walsh, E. J. M., H.; Gorkun, O. V.; Foster, T. J. (2008). Identification of the Staphylococcus aureus MSCRAMM clumping factor B (ClfB) binding site in the $\alpha \mathrm{C}$-domain of human fibrinogen. Microbiology, 154(2), 550-558. doi:10.1099/mic.0.2007/010868-0

60. Forsgren, A. S., J. (1966). "Protein A" from S. aureus. I. Pseudo-immune reaction with human gamma-globulin. J Immunol, 97(6), 822-827.

61. Forsgren, A. Q., P.G. (1974). Effects of Staphylococcal Protein a on Heat Labile Opsonins. J Immunol, 112(3), 1177-1180. 
62. Cramton, S. E., Gerke, C., Schnell, N. F., Nichols, W. W., \& Gotz, F. (1999). The intercellular adhesion (ica) locus is present in Staphylococcus aureus and is required for biofilm formation. Infect Immun, 67(10), 5427-5433.

63. Goss, C. H., \& Muhlebach, M. S. (2011). Review: Staphylococcus aureus and MRSA in cystic fibrosis. J Cyst Fibros, 10(5), 298-306. doi:10.1016/j.jcf.2011.06.002

64. Rolinson, G. N., Stevens, S., Batchelor, F. R., Wood, J. C., \& Chain, E. B. (1960). Bacteriological studies on a new penicillin-BRL. 1241. Lancet, 2(7150), 564-567.

65. Ubukata, K., Nonoguchi, R., Matsuhashi, M., \& Konno, M. (1989). Expression and inducibility in Staphylococcus aureus of the mecA gene, which encodes a methicillin-resistant S. aureus-specific penicillin-binding protein. J Bacteriol, 171(5), 2882-2885.

66. Bloemendaal, A. L., Brouwer, E. C., \& Fluit, A. C. (2010). Methicillin resistance transfer from Staphylocccus epidermidis to methicillin-susceptible Staphylococcus aureus in a patient during antibiotic therapy. PLoS One, 5(7), e11841. doi:10.1371/journal.pone.0011841

67. Apellaniz, G., Valdes, M., Perez, R., Martin, F., Soria, F., Garcia, A., . . . Vicente, T. (1991). Comparison of the effectiveness of various antibiotics in the treatment of methicillin-susceptible Staphylococcus aureus experimental infective endocarditis. J Chemother, 3(2), 91-97.

68. Cantoni, L., Wenger, A., Glauser, M. P., \& Bille, J. (1989). Comparative efficacy of amoxicillin-clavulanate, cloxacillin, and vancomycin against methicillin-sensitive and methicillin-resistant Staphylococcus aureus endocarditis in rats. $J$ Infect Dis, 159(5), 989-993.

69. Chang, S., Sievert, D. M., Hageman, J. C., Boulton, M. L., Tenover, F. C., Downes, F. P., . . . Fridkin, S. K. (2003). Infection with vancomycin-resistant Staphylococcus aureus containing the vanA resistance gene. $N$ Engl J Med, 348(14), 1342-1347. doi:10.1056/NEJMoa025025

70. Razvi, S., Quittell, L., Sewall, A., Quinton, H., Marshall, B., \& Saiman, L. (2009). Respiratory microbiology of patients with cystic fibrosis in the United States, 1995 to 2005. Chest, 136(6), 1554-1560. doi:10.1378/chest.09-0132

71. Rasolomampianina, R., Bailly, X., Fetiarison, R., Rabevohitra, R., Bena, G., Ramaroson, L., . . . Avarre, J. C. (2005). Nitrogen-fixing nodules from rose wood legume trees (Dalbergia spp.) endemic to Madagascar host seven different genera belonging to alpha- and beta-Proteobacteria. Mol Ecol, 14(13), 4135-4146. 
72. Hubner, A., Danganan, C. E., Xun, L., Chakrabarty, A. M., \& Hendrickson, W. (1998). Genes for 2,4,5-trichlorophenoxyacetic acid metabolism in Burkholderia cepacia AC1100: characterization of the tftC and $t f t D$ genes and locations of the $t f t$ operons on multiple replicons. Appl Environ Microbiol, 64(6), 2086-2093.

73. Folsom, B. R., Chapman, P. J., \& Pritchard, P. H. (1990). Phenol and trichloroethylene degradation by Pseudomonas cepacia G4: kinetics and interactions between substrates. Appl Environ Microbiol, 56(5), 1279-1285.

74. Schmidt, S., Blom, J. F., Pernthaler, J., Berg, G., Baldwin, A., Mahenthiralingam, E., \& Eberl, L. (2009). Production of the antifungal compound pyrrolnitrin is quorum sensing-regulated in members of the Burkholderia cepacia complex. Environ Microbiol, 11(6), 1422-1437. doi:10.1111/j.1462-2920.2009.01870.x

75. Jacobs, J. L., Fasi, A. C., Ramette, A., Smith, J. J., Hammerschmidt, R., \& Sundin, G. W. (2008). Identification and onion pathogenicity of Burkholderia cepacia complex isolates from the onion rhizosphere and onion field soil. Appl Environ Microbiol, 74(10), 3121-3129.

76. Blattner, F. R., Plunkett, G., 3rd, Bloch, C. A., Perna, N. T., Burland, V., Riley, M., . . Shao, Y. (1997). The complete genome sequence of Escherichia coli K12. Science, 277(5331), 1453-1462.

77. Holden, M. T., Seth-Smith, H. M., Crossman, L. C., Sebaihia, M., Bentley, S. D., Cerdeno-Tarraga, A. M., . . . Parkhill, J. (2009). The genome of Burkholderia cenocepacia J2315, an epidemic pathogen of cystic fibrosis patients. J Bacteriol, 191(1), 261-277. doi:10.1128/JB.01230-08

78. Laraya-Cuasay, L. L., M.; Huang, N. N. (1977). Pseudomonas cepacia in the respiratory flora of patients with cystic fibrosis (CF). Pediatr. Res., 11, 502.

79. Johnson, W. M., Tyler, S. D., \& Rozee, K. R. (1994). Linkage analysis of geographic and clinical clusters in Pseudomonas cepacia infections by multilocus enzyme electrophoresis and ribotyping. J Clin Microbiol, 32(4), 924930.

80. LiPuma, J. J., Spilker, T., Gill, L. H., Campbell, P. W., 3rd, Liu, L., \& Mahenthiralingam, E. (2001). Disproportionate distribution of Burkholderia cepacia complex species and transmissibility markers in cystic fibrosis. Am J Respir Crit Care Med, 164(1), 92-96. doi:10.1164/ajrccm.164.1.2011153 
81. De Boeck, K., Malfroot, A., Van Schil, L., Lebecque, P., Knoop, C., Govan, J. R., . . V Vandamme, P. (2004). Epidemiology of Burkholderia cepacia complex colonisation in cystic fibrosis patients. Eur Respir J, 23(6), 851-856.

82. Vandamme, P., Holmes, B., Coenye, T., Goris, J., Mahenthiralingam, E., LiPuma, J. J., \& Govan, J. R. (2003). Burkholderia cenocepacia sp. nov.--a new twist to an old story. Res Microbiol, 154(2), 91-96. doi:10.1016/S09232508(03)00026-3

83. Coenye, T., \& LiPuma, J. J. (2002). Multilocus restriction typing: a novel tool for studying global epidemiology of Burkholderia cepacia complex infection in cystic fibrosis. J Infect Dis, 185(10), 1454-1462. doi:10.1086/340279

84. Sajjan, U. S., Corey, M., Karmali, M. A., \& Forstner, J. F. (1992). Binding of Pseudomonas cepacia to normal human intestinal mucin and respiratory mucin from patients with cystic fibrosis. J Clin Invest, 89(2), 648-656.

85. Sajjan, U. S., \& Forstner, J. F. (1993). Role of a 22-kilodalton pilin protein in binding of Pseudomonas cepacia to buccal epithelial cells. Infect Immun, 61(8), 3157-3163.

86. Mahenthiralingam, E., Vandamme, P., Campbell, M. E., Henry, D. A., Gravelle, A. M., Wong, L. T., . . . Speert, D. P. (2001). Infection with Burkholderia cepacia complex genomovars in patients with cystic fibrosis: virulent transmissible strains of genomovar III can replace Burkholderia multivorans. Clin Infect Dis, 33(9), 1469-1475.

87. van Schaik, E. J., Giltner, C. L., Audette, G. F., Keizer, D. W., Bautista, D. L., Slupsky, C. M., . . . Irvin, R. T. (2005). DNA binding: a novel function of Pseudomonas aeruginosa type IV pili. J Bacteriol, 187(4), 1455-1464. doi:10.1128/jb.187.4.1455-1464.2005

88. Lamothe, J., Huynh, K. K., Grinstein, S., \& Valvano, M. A. (2007). Intracellular survival of Burkholderia cenocepacia in macrophages is associated with a delay in the maturation of bacteria-containing vacuoles. Cell Microbiol, 9(1), 40-53.

89. Sajjan, S. U., Carmody, L. A., Gonzalez, C. F., \& LiPuma, J. J. (2008). A type IV secretion system contributes to intracellular survival and replication of Burkholderia cenocepacia. Infect Immun, 76(12), 5447-5455. doi:10.1128/IAI.00451-08

90. Rosales-Reyes, R., Skeldon, A. M., Aubert, D. F., \& Valvano, M. A. (2012). The Type VI secretion system of Burkholderia cenocepacia affects multiple Rho family GTPases disrupting the actin cytoskeleton and the assembly of NADPH oxidase complex in macrophages. Cell Microbiol, 14(2), 255-273. doi:10.1111/j.1462-5822.2011.01716.x 
91. LiPuma, J. J., Dasen, S. E., Nielson, D. W., Stern, R. C., \& Stull, T. L. (1990). Person-to-person transmission of Pseudomonas cepacia between patients with cystic fibrosis. Lancet, 336(8723), 1094-1096.

92. Isles, A., Maclusky, I., Corey, M., Gold, R., Prober, C., Fleming, P., \& Levison, H. (1984). Pseudomonas cepacia infection in cystic fibrosis: an emerging problem. J Pediatr, 104(2), 206-210.

93. Singh, P. K., Schaefer, A. L., Parsek, M. R., Moninger, T. O., Welsh, M. J., \& Greenberg, E. P. (2000). Quorum-sensing signals indicate that cystic fibrosis lungs are infected with bacterial biofilms. Nature, 407(6805), 762-764. doi: $10.1038 / 35037627$

94. Klausen, M., Aaes-Jorgensen, A., Molin, S., \& Tolker-Nielsen, T. (2003). Involvement of bacterial migration in the development of complex multicellular structures in Pseudomonas aeruginosa biofilms. Mol Microbiol, 50(1), 61-68.

95. Guggenheim, M., Shapiro, S., Gmur, R., \& Guggenheim, B. (2001). Spatial arrangements and associative behavior of species in an in vitro oral biofilm model. Appl Environ Microbiol, 67(3), 1343-1350. doi:10.1128/aem.67.3.13431350.2001

96. Nijland, R., Hall, M. J., \& Burgess, J. G. (2010). Dispersal of biofilms by secreted, matrix degrading, bacterial DNase. PLoS One, 5(12), e15668. doi:10.1371/journal.pone.0015668

97. Anwar, H., \& Costerton, J. W. (1990). Enhanced activity of combination of tobramycin and piperacillin for eradication of sessile biofilm cells of Pseudomonas aeruginosa. Antimicrob Agents Chemother, 34(9), 1666-1671.

98. Moskowitz, S. M., Foster, J. M., Emerson, J., \& Burns, J. L. (2004). Clinically feasible biofilm susceptibility assay for isolates of Pseudomonas aeruginosa from patients with cystic fibrosis. J Clin Microbiol, 42(5), 1915-1922.

99. Thurlow, L. R., Hanke, M. L., Fritz, T., Angle, A., Aldrich, A., Williams, S. H., ... Kielian, T. (2011). Staphylococcus aureus biofilms prevent macrophage phagocytosis and attenuate inflammation in vivo. J Immunol, 186(11), 65856596. doi:10.4049/jimmunol.1002794

100. Schommer, N. N., Christner, M., Hentschke, M., Ruckdeschel, K., Aepfelbacher, M., \& Rohde, H. (2011). Staphylococcus epidermidis uses distinct mechanisms of biofilm formation to interfere with phagocytosis and activation of mouse macrophage-like cells 774A.1. Infect Immun, 79(6), 22672276. doi:10.1128/iai.01142-10 
101. Burmolle, M., Webb, J. S., Rao, D., Hansen, L. H., Sorensen, S. J., \& Kjelleberg, S. (2006). Enhanced biofilm formation and increased resistance to antimicrobial agents and bacterial invasion are caused by synergistic interactions in multispecies biofilms. Appl Environ Microbiol, 72(6), 39163923. doi:10.1128/aem.03022-05

102. Lee, K. W., Periasamy, S., Mukherjee, M., Xie, C., Kjelleberg, S., \& Rice, S. A. (2014). Biofilm development and enhanced stress resistance of a model, mixedspecies community biofilm. Isme j, 8(4), 894-907. doi:10.1038/ismej.2013.194

103. Burns, J. L. E., J.; Stapp, J. R.; Yim, D. L.; Krzewinski, J.; Louden, L.; Ramsey, B. W.; Clausen, C. R. (1998). Microbiology of Sputum from Patients at Cystic Fibrosis Centers in the United States. Clin Infect Dis, 27(1), 158-163.

104. Lam, J., Chan, R., Lam, K., \& Costerton, J. W. (1980). Production of mucoid microcolonies by Pseudomonas aeruginosa within infected lungs in cystic fibrosis. Infect Immun, 28(2), 546-556.

105. Tom, S. K., Yau, Y. C., Beaudoin, T., LiPuma, J. J., \& Waters, V. (2015). Effect of High-Dose Antimicrobials on Biofilm Growth of Achromobacter Species Isolated from Cystic Fibrosis Patients. Antimicrob Agents Chemother, 60(1), 650-652. doi:10.1128/aac.02240-15

106. Schwab, U., Abdullah, L. H., Perlmutt, O. S., Albert, D., Davis, C. W., Arnold, R. R., .. Boucher, R. C. (2014). Localization of Burkholderia cepacia complex bacteria in cystic fibrosis lungs and interactions with Pseudomonas aeruginosa in hypoxic mucus. Infect Immun, 82(11), 4729-4745. doi:10.1128/iai.01876-14

107. Vuong, C., Saenz, H. L., Gotz, F., \& Otto, M. (2000). Impact of the agr quorum-sensing system on adherence to polystyrene in Staphylococcus aureus. J Infect Dis, 182(6), 1688-1693. doi:10.1086/317606

108. Di Bonaventura, G., Stepanovic, S., Picciani, C., Pompilio, A., \& Piccolomini, R. (2007). Effect of environmental factors on biofilm formation by clinical Stenotrophomonas maltophilia isolates. Folia Microbiol (Praha), 52(1), 86-90.

109. Hoiby, N., Ciofu, O., \& Bjarnsholt, T. (2010). Pseudomonas aeruginosa biofilms in cystic fibrosis. Future Microbiol, 5(11), 1663-1674. doi:10.2217/fmb.10.125

110. Whiteley, M., Bangera, M. G., Bumgarner, R. E., Parsek, M. R., Teitzel, G. M., Lory, S., \& Greenberg, E. P. (2001). Gene expression in Pseudomonas aeruginosa biofilms. Nature, 413(6858), 860-864. doi:10.1038/35101627

111. Ryan, R. P., Fouhy, Y., Garcia, B. F., Watt, S. A., Niehaus, K., Yang, L., . . . Dow, J. M. (2008). Interspecies signalling via the Stenotrophomonas 
maltophilia diffusible signal factor influences biofilm formation and polymyxin tolerance in Pseudomonas aeruginosa. Mol Microbiol, 68(1), 75-86. doi:10.1111/j.1365-2958.2008.06132.x

112. Tomlin, K. L., Coll, O. P., \& Ceri, H. (2001). Interspecies biofilms of Pseudomonas aeruginosa and Burkholderia cepacia. Can J Microbiol, 47(10), 949-954.

113. Yang, L., Liu, Y., Markussen, T., Hoiby, N., Tolker-Nielsen, T., \& Molin, S. (2011). Pattern differentiation in co-culture biofilms formed by Staphylococcus aureus and Pseudomonas aeruginosa. FEMS Immunol Med Microbiol, 62(3), 339-347. doi:10.1111/j.1574-695X.2011.00820.x

114. O'Toole, G. A. (2011). Microtiter dish biofilm formation assay. J Vis $\operatorname{Exp}(47)$. doi: $10.3791 / 2437$

115. Harrison, J. J., Stremick, C. A., Turner, R. J., Allan, N. D., Olson, M. E., \& Ceri, H. (2010). Microtiter susceptibility testing of microbes growing on peg lids: a miniaturized biofilm model for high-throughput screening. Nat Protoc, 5(7), 1236-1254. doi:10.1038/nprot.2010.71

116. Dubbary, N.; Du, W.; Lane, D.; Pasta, F. (2009). Improved Electrotransformation and Decreased Antibiotic Resistance of the Cystic Fibrosis Pathogen Burkholderia cenocepacia Strain J2315. Appl Environ Microbiol, 74(4), 1095-1102. doi: 10.1128/AEM.02123-09

117. Tang, X. X., Ostedgaard, L. S., Hoegger, M. J., Moninger, T. O., Karp, P. H., McMenimen, J. D., . . Welsh, M. J. (2016). Acidic pH increases airway surface liquid viscosity in cystic fibrosis. J Clin Invest, 126(3), 879-891. doi:10.1172/jci83922

118. Foulston, L., Elsholz, A. K., DeFrancesco, A. S., \& Losick, R. (2014). The extracellular matrix of Staphylococcus aureus biofilms comprises cytoplasmic proteins that associate with the cell surface in response to decreasing $\mathrm{pH}$. MBio, 5(5), e01667-01614. doi:10.1128/mBio.01667-14

119. Sugimoto, S., Iwamoto, T., Takada, K., Okuda, K., Tajima, A., Iwase, T., \& Mizunoe, Y. (2013). Staphylococcus epidermidis Esp degrades specific proteins associated with Staphylococcus aureus biofilm formation and host-pathogen interaction. J Bacteriol, 195(8), 1645-1655. doi:10.1128/jb.01672-12

120. Van Acker, H., Gielis, J., Acke, M., Cools, F., Cos, P., \& Coenye, T. (2016). The Role of Reactive Oxygen Species in Antibiotic-Induced Cell Death in Burkholderia cepacia Complex Bacteria. PLoS One, 11(7), e0159837. doi:10.1371/journal.pone.0159837 
121. Haba, E., Pinazo, A., Jauregui, O., Espuny, M. J., Infante, M. R., \& Manresa, A. (2003). Physicochemical characterization and antimicrobial properties of rhamnolipids produced by Pseudomonas aeruginosa 47T2 NCBIM 40044. Biotechnol Bioeng, 81(3), 316-322. doi:10.1002/bit.10474

122. Davey, M. E., Caiazza, N. C., \& O'Toole, G. A. (2003). Rhamnolipid surfactant production affects biofilm architecture in Pseudomonas aeruginosa PAO1. $J$ Bacteriol, 185(3), 1027-1036.

123. Bartell, J. A., Yen, P., Varga, J. J., Goldberg, J. B., \& Papin, J. A. (2014). Comparative metabolic systems analysis of pathogenic Burkholderia. J Bacteriol, 196(2), 210-226. doi:10.1128/jb.00997-13

124. Hutchison, M. L., Poxton, I. R., \& Govan, J. R. (1998). Burkholderia cepacia produces a hemolysin that is capable of inducing apoptosis and degranulation of mammalian phagocytes. Infect Immun, 66(5), 2033-2039.

125. Mashburn, L. M., Jett, A. M., Akins, D. R., \& Whiteley, M. (2005). Staphylococcus aureus serves as an iron source for Pseudomonas aeruginosa during in vivo coculture. $J$ Bacteriol, 187(2), 554-566. doi:10.1128/JB.187.2.554-566.2005

126. Darling, P., Chan, M., Cox, A. D., \& Sokol, P. A. (1998). Siderophore production by cystic fibrosis isolates of Burkholderia cepacia. Infect Immun, 66(2), 874-877.

127. Sokol, P. A., Darling, P., Lewenza, S., Corbett, C. R., \& Kooi, C. D. (2000). Identification of a siderophore receptor required for ferric ornibactin uptake in Burkholderia cepacia. Infect Immun, 68(12), 6554-6560.

128. Sokol, P. A., Darling, P., Woods, D. E., Mahenthiralingam, E., \& Kooi, C. (1999). Role of ornibactin biosynthesis in the virulence of Burkholderia cepacia: characterization of $p v d A$, the gene encoding L-ornithine $\mathrm{N}(5)$ oxygenase. Infect Immun, 67(9), 4443-4455.

129. Visser, M. B., Majumdar, S., Hani, E., \& Sokol, P. A. (2004). Importance of the ornibactin and pyochelin siderophore transport systems in Burkholderia cenocepacia lung infections. Infect Immun, 72(5), 2850-2857.

130. Rayner, C., \& Munckhof, W. J. (2005). Antibiotics currently used in the treatment of infections caused by Staphylococcus aureus. Intern Med J, 35 Suppl 2, S3-16. doi:10.1111/j.1444-0903.2005.00976.x 


\section{Appendix}

\section{Supplementary Table}

\section{Supplementary Figure}


I. Supplementary Table 
Supplementary Table 1. Description of incubation times and colony morphology by strain.

\begin{tabular}{|c|c|c|}
\hline Strain & Colony Morphology & Incubation Time \\
\hline $\begin{array}{c}\text { A. xylosoxidans } \\
\text { AU19284 }\end{array}$ & Small colonies, white & $48 \mathrm{~h}$ \\
\hline P. aeruginosa C3719 & Large colonies, tinged pink & $24-48 \mathrm{~h}$ \\
\hline S. maltophilia K279a & $\begin{array}{c}\text { Largest colonies, dark } \\
\text { yellow }\end{array}$ & $24 \mathrm{~h}$ \\
\hline S. aureus NRS77 & Large colonies, white & $24 \mathrm{~h}$ \\
\hline B. cenocepacia J2315 & $\begin{array}{c}\text { Smallest colonies, tan, } \\
\text { produce a brown pigment }\end{array}$ & At least $48 \mathrm{~h}$ \\
\hline
\end{tabular}


II. Supplementary Figure 
A
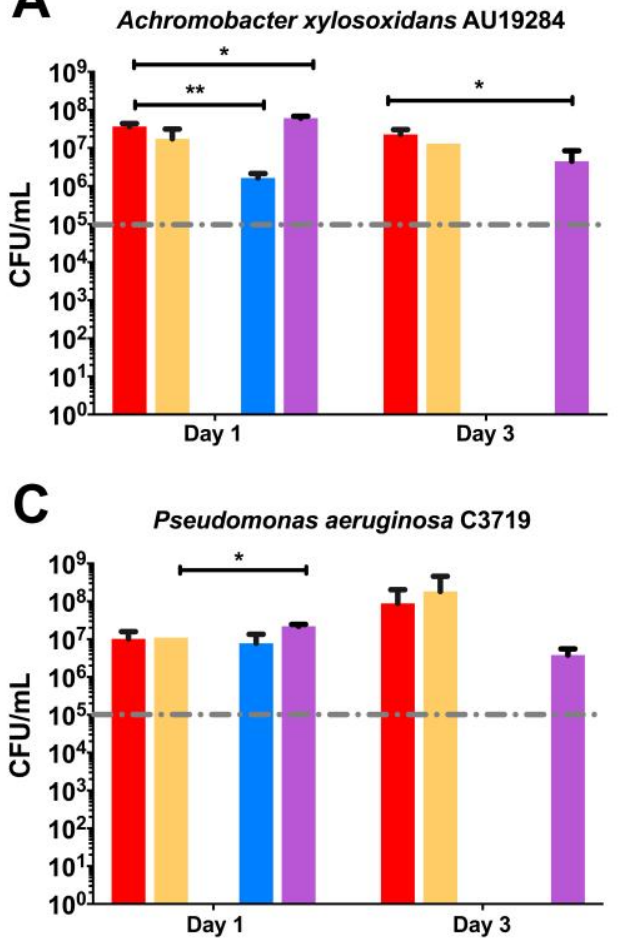

B

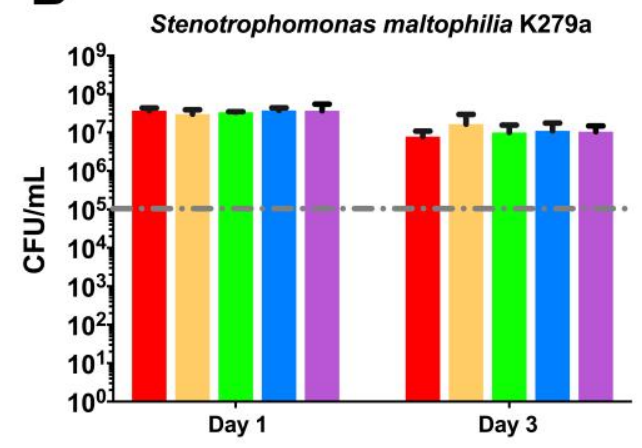

A. xylosoxidans AU19284

P. aeruginosa C3719

S. maltophilia K279a

S. aureus NRS77

B. cenocepacia J2315

Supplementary Figure 1. Co-culture biofilm formation of common CF pathogens. Viable cell counts of A. xylosoxidans AU19284 (A), S. maltophilia K279a (B), and P. aeruginosa C3719 (C) biofilm grown were compared when grown in with A. xylosoxidans (red), P. aeruginosa C3719 (orange), S. maltophilia K279a (green), S. aureus NRS77 (blue), or B. cenocepacia J2315 (purple). Presence of " $X$ " indicates contamination, and therefore exclusion of data. Absence of a bar indicates the CFU/mL is below the limit of detection, which was $10^{5}$ for this experiment. One-way ANOVA analysis with Tukey's multiple comparison test was used to determine significance $(* \mathrm{p}<0.05, * * \mathrm{p}<0.01)$.

The ability of S. maltophilia K279a to form biofilm was not affected by the presence of the tested strains. There was no significant difference in S. maltophilia K279a biofilm formation in monoculture compared to co-culture conditions on both day one and day three of biofilm formation. On the other hand, the presence of S. maltophilia K279a in co-culture reduced biofilm formation below the limit of detection in both $A$. xylosoxidans AU19284 and $P$. aeruginosa C3719. The biofilm formation of $A$. xylosoxidans AU19284 in co-culture with B. cenocepacia J2315 was significantly greater than monoculture on day one, but was significantly lower than monoculture on day three. Interestingly, the presence of $S$. aureus NRS77 significantly inhibits A. xylosoxidans AU19284 biofilm formation on day one, and reduces biofilm below the limit of detection on day three. $P$. aeruginosa C3719 biofilm was significantly increased due to the presence of $B$. cenocepacia $\mathrm{J} 2315$ on day one, but biofilm formation on day three in this co-culture condition was not statistically significant in comparison to monoculture. Repeated experimentation over a longer period of time or with a lower limit of detection would further elucidate the interactions between some of these isolates in co-culture conditions. 